\title{
On recovering continuum topology from a causal set
}

\author{
Seth Major ${ }^{\text {a) }}$ \\ Department of Physics, Hamilton College, Clinton, New York 13323 \\ David Rideout ${ }^{\text {b) }}$ \\ Blackett Laboratory, Imperial College, London SW7 2AZ, United Kingdom \\ Sumati Surya ${ }^{\mathrm{c})}$ \\ Raman Research Institute, Bangalore 560 080, India
}

(Received 7 June 2006; accepted 19 December 2006; published online 6 March 2007)

\begin{abstract}
An important question that discrete approaches to quantum gravity must address is how continuum features of space-time can be recovered from the discrete substructure. Here, we examine this question within the causal set approach to quantum gravity, where the substructure replacing the space-time continuum is a locally finite partial order. A new topology on causal sets using "thickened antichains" is constructed. This topology is then used to recover the homology of a globally hyperbolic space-time from a causal set which faithfully embeds into it at sufficiently high sprinkling density. This implies a discrete-continuum correspondence which lends support to the fundamental conjecture or "Hauptvermutung" of causal set theory. (C) 2007 American Institute of Physics. [DOI: 10.1063/1.2435599]
\end{abstract}

\section{INTRODUCTION}

Space-time discretization is a common calculational device used to regularize background dependent physics. Typically, the discretization is topologically trivial, with the space-time continuum replaced by a lattice which is regular in a preferred reference frame. Physical results are then obtained by taking this cutoff to zero. Two important issues which arise as a result of a naive space-time discretization are already apparent in quantum field theory on Minkowski space-time. The first is the breaking of Poincaré invariance, and the second, the loss of global topological information. Since discreteness is used only as a calculational tool, these issues only pose practical limits on the discretization, since relevant physics is, by and large, recovered in the continuum limit.

However, both issues assume a more fundamental role in discrete approaches to quantum gravity, in which the continuum is taken to arise as an approximation rather than as a limit. Instead of being a means to regulate the theory, space-time discreteness is taken to be fundamental, much like the atomicity of an apparently continuous fluid. The choice of the discrete building blocks in a given approach to quantum gravity then determines the manner in which these two issues manifest themselves.

In many discrete approaches to quantum gravity, local Lorentz invariance is explicitly broken, and much recent work has been devoted to quantifying such violations. For instance, in the case of modified dispersion relations, threshold analyses demonstrate that current astrophysical observations place severe constraints on cubic modifications. ${ }^{1}$ Significantly, in the causal set approach to quantum gravity, no such violation occurs. This unique property arises from the fact that the continuum approximation of the theory obtains from a random process. ${ }^{2}$

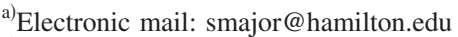

${ }^{b}$ Electronic mail: dprideout@gmail.com

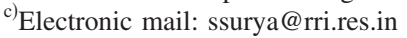


In this work, we address the question of how to recover continuum topology from the discrete substructure within the framework of causal set quantum gravity. ${ }^{3,4}$ In this approach, the spacetime continuum is replaced by a causal set or causet which is a locally finite partially ordered set. The continuum approximation of the theory obtains from a "faithful embedding" $\Phi: C \rightarrow M$ of the causet $C$ to a space-time $(M, g)$ at space-time density $\rho$, i.e., $\Phi(C) \subset M$ is a high probability random (Poisson) distribution of points on $M$ at density $\rho$ such that the order relation in $C$ is mapped to a causal relation in $(M, g)$. A causet embedded in $(M, g)$ thus resembles a random space-time lattice, where the directed links between two points indicate a causal relation.

A key conjecture of causal set theory, the "Hauptvermutung," states that the continuum approximation of a causet is unique up to an approximate isometry; thus, if $\Phi: C \rightarrow(M, g)$ is a faithful embedding at density $\rho$, then $(M, g)$ is unique up to isomorphisms at "scales above $\rho^{-1}$." While this has been proven rigorously in the infinitely fine grained limit, $G \hbar \rightarrow 0,{ }^{5}$ there is evidence from calculations of dimensions and geodesic distance that provide support for the conjecture in the finite case. ${ }^{6,7}$ For example, for a causet $C$ which faithfully embeds into $d$-dimensional Minkowski space-time $\mathbb{M}^{d}$, the "Myrheim-Meyer" dimension of an interval in $C$ gives a good estimate of the continuum dimension. ${ }^{6}$ Thus, if a causet $C$ embeds faithfully into $\mathbb{M}^{d_{1}}$ and $\mathbb{M}^{d_{2}}$ at the same sprinkling density, then $d_{1} \sim d_{2}$.

It is therefore of interest to seek a correspondence between the continuum topology and an appropriately defined topology on the causal set. Such a correspondence would then imply that if a causet faithfully embeds into two space-times with topologies $M_{1}$ and $M_{2}$, then there is an approximate homeomorphism $M_{1} \sim M_{2}$. This would also imply that $M_{1}$ and $M_{2}$ are homologous at scales larger than $\rho^{-1}$. In this work we make considerable progress in establishing a correspondence between the homology of thickened antichains which are special subcausets of $C$ and that of a globally hyperbolic space-time into which it faithfully embeds. Under certain assumptions, this implies a homological version of the Hauptvermutung.

The random nature of the sprinkled causal set makes the task of finding a correspondence fairly nontrivial. In approaches using simplicial methods such as dynamical triangulations or spin foams, the discrete structure can be taken to be a triangulation of the given manifold, which though nondiffeomorphic to the continuum, by construction, carries all continuum homological information. ${ }^{8}$ Conversely, an abstract simplicial complex is associated with a given manifold only if it can be mapped bijectively to a triangulation of that space. In causal set theory, however, this connection is apparently more tenuous, since the discretization does not preserve continuum topological information in an obvious way.

However, a nontrivial partial order does possess sufficient structure compared to the unordered set of points on a lattice and hence admits nontrivial topologies. ${ }^{9,10}$ For example, a chain complex on $C$ is constructed by mapping every $k$-element chain, or totally ordered subset, to a $k$-simplex, while the interval topology is constructed from sets which are analogs of the Alexandrov intervals in a space-time. ${ }^{10}$ Indeed, it has been recently shown that a globally hyperbolic space-time is a so-called bicontinuous poset (in particular, this means that the poset is not locally finite, and hence not a causal set according to our definition) whose interval topology is the same as the manifold topology. ${ }^{11}$ Thus, partially ordered sets admit very rich topological structures.

For a locally finite causal set, however, it is unclear how these topologies relate to the continuum topology. An important first step is to realize that the topology of the continuum approximation is too rich and contains "irrelevant" information on scales below the discreteness scale $\rho^{-1}$. Thus, the assumption of a fundamental discreteness is incompatible with the requirement that there exists a strict homeomorphism between the causal set topology and the continuum topology. Physical significance cannot be attributed to continuum structures of characteristic size smaller than $\rho^{-1}$ and hence only macroscopic topological information, such as homology or homotopy at scales $\gg \rho^{-1}$ can be considered relevant to the causet. This observation is true of any finitary topology. ${ }^{12}$

In this work we provide a prescription for constructing a map between discrete and continuum homologies for the class of causal sets that faithfully embed into globally hyperbolic space-times. The simplicial complex we construct for the causal set uses the discrete analog of a Cauchy 
hypersurface, i.e., an inextendible antichain or a maximal set of unordered points in the causal set. ${ }^{13}$ While an inextendible antichain, being endowed with only the trivial topology, does not itself suffice to capture any continuum topological information, it does inherit a nontrivial structure from its embedding in the larger causal set to which it belongs. As in Ref. 13, starting with an inextendible antichain $A$ we define the discrete analog of a space-time volume "thickened" Cauchy hypersurface or thickened antichain

$$
\mathrm{T}_{\mathrm{n}}(A) \equiv\{p||(\{p\} \sqcup \operatorname{Past}(p)) \cap(\{A\} \sqcup \operatorname{Fut}(A)) \mid \leqslant \mathrm{n}\},
$$

where $\mathrm{n} \in \mathbb{N},|X|$ is the cardinality of a set $X$ and $\operatorname{Past}(p)$ and $\operatorname{Fut}(p)$ are the past and future of $p$, respectively. For finite $\mathrm{n}$, every element in $\mathrm{T}_{\mathrm{n}}(A)$ has a finite past in $\mathrm{T}_{\mathrm{n}}(A)$, and hence one can find the set of maximal or future-most points in $\mathrm{T}_{\mathrm{n}}(A)$. A nerve simplicial complex is then constructed from the shadows of the past sets of these maximal elements onto $A$, details of which will be described in the following sections. Nontrivial overlaps of these shadows imply a nontrivial simplicial complex from which one can extract homological information.

On the face of it, there is nothing to suggest that the above construction is more natural than the chain or interval topologies. However, preliminary numerical simulations in $1+1$ dimensions suggest the existence of a robust correspondence between this causal set topology and the continuum topology. ${ }^{14}$ In this work, using purely analytical tools, we show that the continuum analogue of this construction in a globally hyperbolic space-time does indeed yield a nerve simplicial complex which is not only homologous but homotopic to the space-time. Moreover, we show that there exists an isomorphism between the homology groups of the discrete and continuum nerves for causets which faithfully embed into globally hyperbolic space-times.

We begin with some basic definitions in Sec. II. We then construct the nerve simplicial complex for the discrete and the continuum cases in Sec. III. In Sec. IV, we prove an important continuum result. Namely, we show that the continuum nerve is a simplicial complex which is homotopic to the space-time manifold $M$ for a class of "volume thickenings" of a Cauchy surface. We make crucial use of a theorem due to De Rham and Weil on the nerve of a locally finite convex cover. Finally, we prove our main result in Sec. V, i.e., we show for a class of inextendible antichains in a faithful embedding that with high probability the order-theoretic nerve simplicial complex is homologically equivalent to the manifold for sufficiently high sprinkling densities $\rho$. We summarize our results in Sec. VI and conclude with a discussion of some of the open questions. Since this work is heavy with notation, the Appendix provides a table of the symbols used and their definitions.

\section{PRELIMINARIES}

Here we set down some definitions and notations that we will need.

\section{A. The causal set}

A causal set $C$ is a set with an order relation $<$ which is (i) transitive, i.e., $x<y$ and $y$ $<z \Rightarrow x<z$ ), (ii) irreflexive, i.e., $x \nless x$, and (iii) locally finite, i.e., $|\operatorname{Past}(x) \cap \operatorname{Fut}(y)|<\infty$ for any $x, y, z \in C$, where $\operatorname{Past}(y)=\{x \mid x<y\}$, Fut $(y)=\{x \mid y<x\}$, and $|A|$ denotes set cardinality.

These discrete analogs of the causal future/past sets of the continuum do not include $x$ because of the irreflexive condition. Since such an inclusion will find use in our constructions, we define the inclusive future and past sets as $\operatorname{IFut}(x) \equiv x \cup \operatorname{Fut}(x)$ and $\operatorname{IPast} \equiv x \cup \operatorname{Past}(x)$, respectively.

\section{B. Causal relations in the continuum}

We will use the notation and results from Ref. $15 . I^{ \pm}(p)$ denotes the chronological future/past of an event $p$ and $\mathrm{J}^{ \pm}(p)$ its causal future/past. We will refer to the region spacelike to $p$ by $S(p)$ $\equiv M \backslash\left(\mathrm{J}^{+}(p) \cup \mathrm{J}^{-}(p)\right)$. The Alexandrov interval is defined to be the open set $\mathrm{I}(p, q)$ $\equiv \mathrm{I}^{+}(p) \cap \mathrm{I}^{-}(q)$. The generalization of these definitions for sets is straightforward, as is the notation. In a globally hyperbolic space-time $(M, g), \overline{\mathrm{I}(p, q)}$ is compact for any $p, q \in M$. For $p, q$ 
$\in M$ we will use the notations $p<<q, p<q$, and $p \rightarrow q$ to denote chronological, causal, and null relations, respectively. We will find use for the result $p<<q, q<r \Rightarrow p<<r$.

Let $F$ be a function which assigns to each event $x$ in $M$ an open set $F(x) \subset M$. Then $F$ is said to be inner continuous if for any $x$ and any compact $K \subset F(x)$, there exists a neighborhood $U$ of $x$ with $K \subset F(y)$ for all $y \in U, F$ is said to be outer continuous if for any $x$ and any compact set $K \subset M \backslash \overline{F(x)}$, there exists a neighborhood $U$ of $x$ with $K \subset M \backslash \overline{F(y)} \forall y \in U$.

In a time-orientable distinguishing space-time $(M, g)$, the sets $\mathrm{I}^{+}(x)$ and $\mathrm{I}^{-}(x)$ are inner continuous, ${ }^{16}$ but need not be outer continuous. A space-time is said to be causally continuous if for all events $x \in M, \mathrm{I}^{+}(x)$ and $\mathrm{I}^{-}(x)$ are outer continuous. A globally hyperbolic space-time is causally continuous.

A convex normal neighborhood (CNN) $\mathrm{N} \subset M$ is an open set such that for any $p \in \mathrm{N}$, there exists a map $\exp _{p}$ from the tangent space $T_{p} \mathrm{~N}$ to $\mathrm{N}$ which is a diffeomorphism. An important feature of $\mathrm{N}$ is that any two points in $\mathrm{N}$ are joined by a unique geodesic which lies entirely in $\mathrm{N}$.

\section{Some definitions from Riemannian geometry}

In a Riemannian space $(\Sigma, h)$, the distance function $d(x, y)$ between two points on $\Sigma$ is the length of the shortest path between $x$ and $y$. A curve between $x, y$ is a segment if its length is $d(x, y)$. The convexity radius at a $p \in \Sigma$ is the largest $\mathfrak{r}_{p}$ such that the distance function $d(x, p)$ is a convex function on the open ball $B\left(p, \mathfrak{r}_{p}\right)$ and any two points in $B\left(p, \mathfrak{r}_{p}\right)$ are joined by a unique segment lying entirely in $B\left(p, \mathfrak{r}_{p}\right)$. The convexity radius $\mathfrak{r}$ of $\Sigma$ is given by the infimum of $\mathfrak{r}_{p}$ for all $p \in \Sigma$. Thus, if $d(p, q)<\mathfrak{r}$ for any $p, q \in \Sigma$, then there exists a unique geodesic from $p$ to $q$ of arclength $<\mathfrak{r}$. An open set $\mathrm{N} \subset \Sigma$ is said to be convex with respect to $\mathfrak{r}$ if for any $p, q \in \mathrm{N}$, the (unique) geodesic between them of arclength $<\mathfrak{r}$ lies entirely in N. The intersection of two convex sets is then also convex with respect to $\mathfrak{r}$. A convex cover of $\Sigma$ with respect to $\mathfrak{r}$ is a locally finite cover of $\Sigma$ with each element a convex set with respect to $r$. $\operatorname{diam}(\mathrm{N})$ is the diameter of an open set $\mathrm{N}$ which is the length of the longest geodesic between any two points in $\mathrm{N} .{ }^{17}$

\section{THE NERVE SIMPLICIAL COMPLEX}

We begin by constructing the nerve simplicial complex for an arbitrary causal set. Let $C$ be a causal set with $A \subset C$ an inextendible antichain, and $\mathrm{T}_{\mathrm{n}}(A)$ an associated thickened antichain for some $\mathrm{n} \geqslant 0$ as defined in Eq. (1). Let $\mathrm{M}$ be the set of maximal or future-most elements in $\mathrm{T}_{\mathrm{n}}(A)$ and $P_{i} \equiv \operatorname{IPast}\left(m_{i}\right) \cap \operatorname{IFut}(A), m_{i} \in \mathrm{M}$. The collection $\mathcal{P} \equiv\left\{P_{i}\right\}$ is a covering of $\mathrm{T}_{\mathrm{n}}(A)$, i.e., $\cup_{i} P_{i}$ $=\mathrm{T}_{\mathrm{n}}(A)$, since $P_{i} \subset \mathrm{T}_{\mathrm{n}}(A)$ for all $i$ and any $x \in \mathrm{T}_{\mathrm{n}}(A)$ belongs to the inclusive past of some maximal element of $\mathrm{T}_{\mathrm{n}}(A)$. For each $P_{i}$, define the shadow sets $A_{i} \equiv P_{i} \cap A \subset A$. Again, since $A_{i} \subset \mathrm{T}_{\mathrm{n}}(A)$ for all $i$, and any $a \in A$ lies to the inclusive past of a maximal element of $\mathrm{T}_{\mathrm{n}}(A)$, the collection $\mathcal{A} \equiv\left\{A_{i}\right\}$ covers $A$.

The nerve simplicial complex $\mathcal{N}(\mathcal{A})$ of $A$ is then constructed by mapping each $A_{i}$ to a vertex, every nonvanishing intersection $A_{i_{0}} \cap A_{i_{1}} \neq \emptyset$ to a 1 -simplex, and in general, every nonvanishing intersection $A_{i_{0}} \cap A_{i_{1}} \cdots A_{i_{k}} \neq \emptyset$ to a $k$-simplex. ${ }^{18}$ This construction is illustrated in Fig. 1 . The nerve simplicial complex $\mathcal{N}(P)$ of $\mathrm{T}_{\mathrm{n}}(A)$ can be similarly constructed.

We now show that there exists a map $\Psi^{*}: \mathcal{N}(\mathcal{P}) \rightarrow \mathcal{N}(\mathcal{A})$ which is a bijection. Let us define the projection map $\Psi: \mathcal{P} \rightarrow \mathcal{A}$, i.e., $\Psi\left(P_{i}\right)=P_{i} \cap A=A_{i}$. By definition, $\Psi$ is onto. However, for an arbitrary causal set, it is possible that $\Psi$ is not one-to-one: $P_{i} \cap A=P_{j} \cap A$ need not imply that $i$ $=j$, so that the shadows $A_{i}$ and $A_{j}$ are identical as subsets of $A$. Strictly speaking, then, the collection $\mathcal{A}=\left\{A_{i}\right\}$ is a cover of $A$, only if such "redundant" subsets are removed from it. Let us, however, drop the requirement that $\mathcal{A}$ be a cover of $A$ and retain these redundant elements. Since now every $A_{i}$ comes from a unique $P_{i}, \Psi$ is a bijection. Moreover, by set inclusion, any nonvanishing intersection $A_{i_{0} \ldots i_{k}} \equiv A_{i_{0}} \cap A_{i_{2}} \cdots \cap A_{i_{k}} \neq \emptyset$ has an associated nonvanishing $P_{i_{0} \ldots i_{k}}$ $\equiv P_{i_{0}} \cap P_{i_{2}} \cdots P_{i_{k}} \neq \emptyset$. Hence, a $k$-simplex in $\mathcal{N}(\mathcal{A})$ maps to a $k$-simplex in $\mathcal{N}(\mathcal{P})$, i.e., the map $\Psi^{*}: \mathcal{N}(\mathcal{A}) \rightarrow \mathcal{N}(\mathcal{P})$ is one-to-one. Moreover, if $P_{i_{0} \ldots i_{k}} \neq \emptyset$, then $P_{i_{0} \ldots i_{k}} \cap A \neq \emptyset$ : every $x \in P_{i_{0} \ldots i_{k}}$ has a nonempty inclusive past in $\mathrm{T}_{\mathrm{n}}(A), \operatorname{IPast}(x) \cap \operatorname{IFut}(A)$, which, by transitivity, is a subset of $P_{i_{0} \ldots i_{k}}$ 


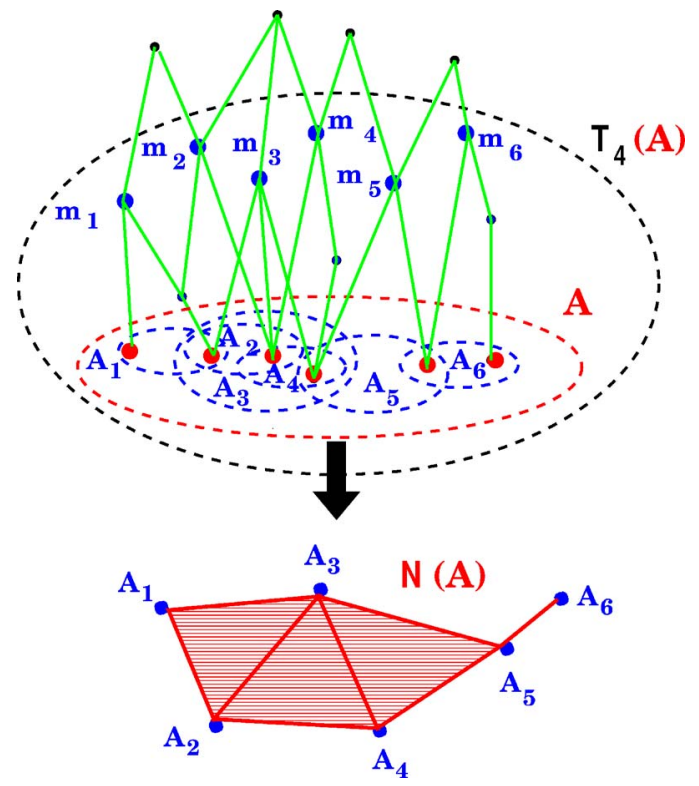

FIG. 1. (Color online) Constructing a nerve simplicial complex $\mathcal{N}(\mathcal{A})$ from an inextendible antichain $A$.

which means that $\exists a \in P_{i_{0} \ldots i_{k}}$ such that $a \in A$. Or, $P_{i_{0} \ldots i_{k}} \cap A=\left(P_{i_{0}} \cap A\right) \cap\left(P_{i_{1}} \cap A\right) \cap \cdots\left(P_{i_{k}} \cap A\right)$ $=A_{i_{0} \ldots i_{k}} \neq \emptyset$. Thus, a $k$-simplex in $\mathcal{N}(\mathcal{P})$ maps to a $k$-simplex in $\mathcal{N}(\mathcal{A})$, which means that $\left(\Psi^{*}\right)^{-1}$ is one-to-one, thus establishing $\Psi^{*}$ as a bijection.

Preliminary numerical investigations for causets which faithfully embed into $1+1$ dimensional space-times show that $\mathcal{N}(\mathcal{A})$ is homologically equivalent to the continuum for a large range of values of $n .{ }^{14}$ In Fig. 2 we show the results of numerical simulations for a causal set embedded into an $M=S^{1} \times \mathbb{R}$ cylinder space-time. In the continuum, the nonvanishing Betti numbers are $b_{0}(M)=b_{1}(M)=1$, and there is no torsion. For $\mathrm{n} \leqslant 15, \mathrm{~T}_{\mathrm{n}}(A)$ splits into disconnected pieces, so that $b_{0}(\mathrm{~T})>b_{0}(M)$. Similarly, for $\mathrm{n} \geqslant 504,\left\{b_{i}(\mathrm{~T})\right\} \neq\left\{b_{i}(M)\right\}$. However, there exist a large range of thickenings, $16 \leqslant \mathrm{n} \leqslant 503$, for which $\left\{b_{i}(\mathrm{~T})\right\}=\left\{b_{i}(M)\right\}$. Details will be discussed elsewhere, ${ }^{14}$ but for now, it serves to justify our use of the discrete simplicial complex $\mathcal{N}(\mathcal{A})$ as the appropriate starting point of our subsequent analytical investigations.

The first task is to find an appropriate continuum analog of the nerve $\mathcal{N}(\mathcal{A})$ and to ask how it is related to the space-time topology. For a globally hyperbolic space-time $(M, g)$, we first note that a Cauchy hypersurface $\Sigma$ is an appropriate continuum analog of the inextendible antichain $A$ since every point in $(M, g)$ is either to the future or past of $\Sigma .^{13}$ The continuum analog of the thickened antichain $T_{n}(A)$ can be obtained by correlating the number of elements to the space-time volume. Starting with a Cauchy hypersurface $\Sigma$, one thus obtains a "volume thickened" region either to the past or future of $\Sigma$. We now describe this construction in detail.

Define the volume function $\vee$ on $M$ with respect to a Cauchy hypersurface $\Sigma$ :

$$
\mathrm{v}(p)= \begin{cases}\operatorname{vol}(\mathrm{I}(\Sigma, p)), & p \in \mathrm{I}^{+}(\Sigma) \\ -\operatorname{vol}(\mathrm{I}(p, \Sigma)), & p \in \mathrm{I}^{-}(\Sigma) \\ 0, & p \in \Sigma,\end{cases}
$$

where $\operatorname{vol}(X)$ denotes the space-time volume of a region $X \subset M$. Let $\xi^{a}$ denote a continuous future-directed timelike vector field on $(M, g)$ and $\xi(s)$ an associated integral curve of $\xi^{a}$, where $s \in \Sigma$. For a globally hyperbolic space-time $(M, g)$ with compact Cauchy surface $\Sigma$ we show the following.

Lemma 1: $\mathrm{v}(p)$ is a monotonically increasing (decreasing) continuous function along the integral curves $\xi(s)$ of $\xi^{a}$ to the causal future (past) of $\Sigma$, where $s \in \Sigma$. 

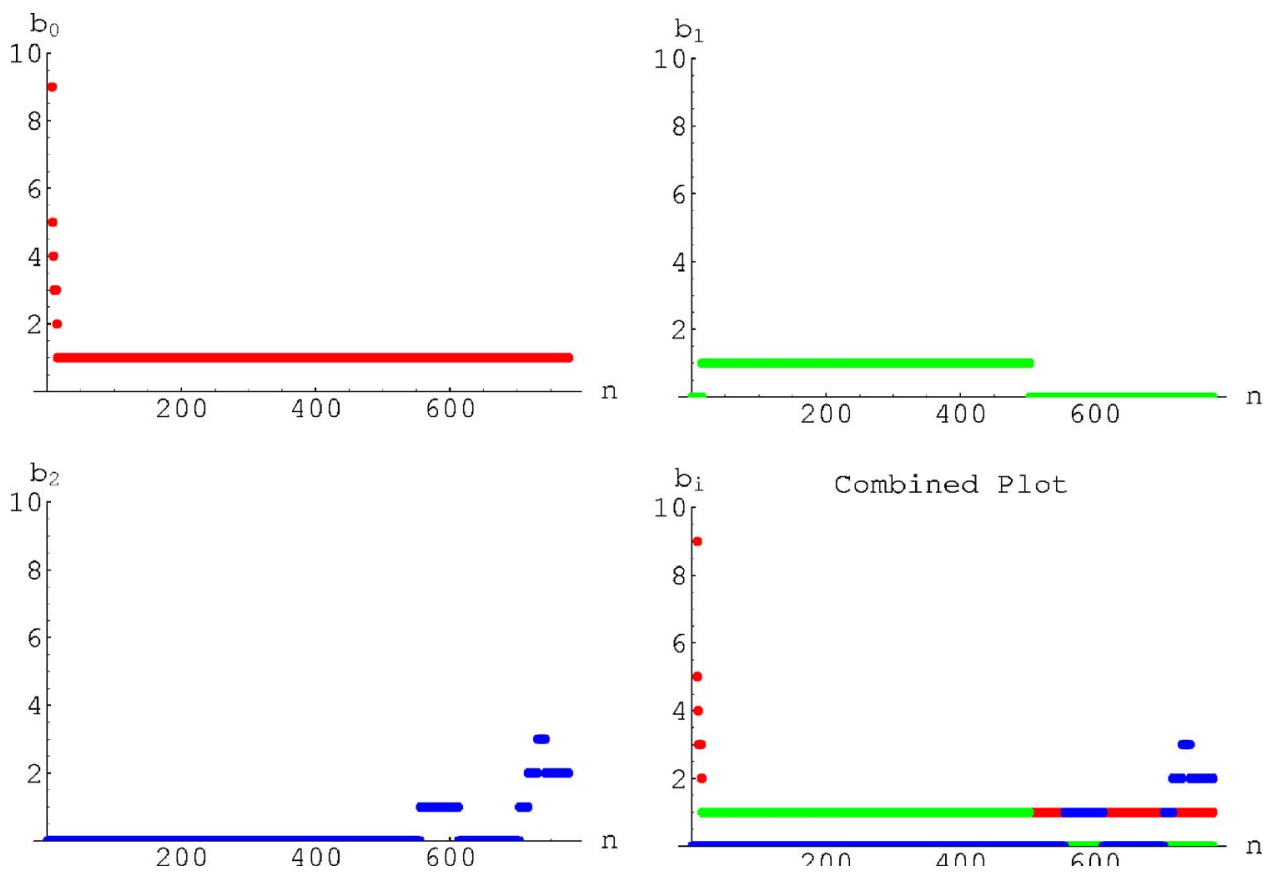

FIG. 2. (Color online) The first three plots show the first three Betti numbers $b_{i}$ vs thickening volume $n$ for a causet sprinkled onto a region of the cylinder space-time $S^{1} \times \mathbb{R}$, whose nonvanishing Betti numbers are $b_{0}=1, b_{1}=1$. Superimposing these plots then helps us determine the range of thickenings $(16 \leqslant n \leqslant 503)$ for which the thickened antichain homology matches that of the continuum. (The torsion vanishes uniformly and is hence not plotted.)

Proof: Monotonicity is immediate. Let $\lambda$ be the parameter along the integral curves $\xi_{\lambda}(s)$. Let $q, r$ be two points on $\xi_{\lambda}(s)$ which lie in $\mathrm{I}^{+}(\Sigma)$ such that $r \in \mathrm{I}^{+}(q)$. The interval $\mathrm{I}(q, r)$ is nonempty and with compact closure, since $(M, g)$ is globally hyperbolic. Hence $\operatorname{vol}(\mathrm{I}(q, r))$ is finite and nonzero. Since $\mathrm{I}(q, r) \subset \mathrm{I}(\Sigma, r)$, but $\mathrm{I}(q, r) \nsubseteq \mathrm{I}(\Sigma, q), \mathrm{v}(r) \geqslant \mathrm{V}(q)+\operatorname{vol}(\mathrm{I}(q, r))$, or $\mathrm{v}(r)>\mathrm{V}(q)$. Since there exists a $\lambda_{1}<\lambda_{2}$ such that $q=\xi_{\lambda_{1}}(s)$ and $r=\xi_{\lambda_{2}}(s)$, we see that $\mathrm{v}\left(\lambda_{1}\right) \equiv \mathrm{v}(q)<\mathrm{v}\left(\lambda_{2}\right) \equiv \mathrm{v}(r)$ for any $\lambda_{1}<\lambda_{2}$.

Next, we show that $\mathrm{v}$ is a continuous function on $\xi(s)$. For a given $s \in \Sigma$, the points in $\xi(s)$ can simply be labeled by the parameter $\lambda$. Let $\lambda_{0}$ be such that $\xi_{\lambda_{0}}(s)$ lies in $\mathrm{I}^{+}(s)$. Define $\overline{\mathrm{I}}_{ \pm} \equiv \mathrm{Lt}_{\epsilon \rightarrow 0} \mathrm{l}\left(\Sigma, \lambda_{0} \pm \epsilon\right)$ for $\epsilon>0$ and $\mathrm{v}_{ \pm} \equiv \operatorname{vol}\left(\overline{\mathrm{I}_{ \pm}}\right)=\operatorname{vol}\left(\mathrm{Lt}_{\epsilon \rightarrow 0} \mathrm{l}\left(\Sigma, \lambda_{0} \pm \epsilon\right)\right)=\mathrm{Lt}_{\epsilon \rightarrow 0} \mathrm{v}\left(\lambda_{0} \pm \epsilon\right)$. Monotonicity implies that $\mathrm{v}\left(\lambda_{0}-\epsilon\right)<\mathrm{v}\left(\lambda_{0}\right)<\mathrm{v}\left(\lambda_{0}+\epsilon\right)$ for $\epsilon>0$ and hence $\mathrm{v}_{-} \leqslant \mathrm{v}\left(\lambda_{0}\right) \leqslant \mathrm{v}_{+}$.

Let $\mathrm{v}_{-}<\mathrm{v}\left(\lambda_{0}\right)$ be a strict inequality. This means that $\mathrm{I}\left(\Sigma, \lambda_{0}\right)$ contains a set $S$ of finite volume such that $S \nsubseteq \mathrm{I}\left(\Sigma, \lambda_{0}-\epsilon\right)$ for any $\epsilon>0$. For any compact subset $K \subset S$, this implies that $K \subset \mathrm{I}^{-}\left(\lambda_{0}\right)$, but $K \nsubseteq \mathrm{I}\left(\Sigma, \lambda_{0}-\epsilon\right) \forall \epsilon>0$. Using the set decomposition $\mathrm{I}^{-}\left(\lambda_{0}-\epsilon\right)=\mathrm{I}\left(\Sigma, \lambda_{0}-\epsilon\right) \sqcup\left(\mathrm{I}^{-}\left(\lambda_{0}\right.\right.$ $-\epsilon) \cap \Sigma) \sqcup \mathrm{I}^{-}\left(\left(\mathrm{I}^{-}\left(\lambda_{0}-\epsilon\right) \cap \Sigma\right)\right)$, we see that since $K$ neither is in or to the past of $\Sigma, K \nsubseteq \mathrm{I}^{-}\left(\lambda_{0}-\epsilon\right)$. Thus, even though $K \subset \mathrm{I}^{-}\left(\lambda_{0}\right)$, every neighborhood of $\lambda_{0}$ contains a $p \in \mathrm{I}^{-}\left(\lambda_{0}\right)$ such that $K \nsubseteq \mathrm{I}^{-}(p)$, i.e., $I^{-}\left(\lambda_{0}\right)$ is not inner continuous, which is a contradiction.

Now let $v_{+}>v\left(\lambda_{0}\right)$ be a strict inequality. This means that $I_{+}$is not simply the closure of $\mathrm{I}^{-}\left(\Sigma, \lambda_{0}\right)$, and hence there exists an $S \subset \operatorname{int}\left(\mathrm{I}_{+}\right)$of finite volume such that $S \Phi \overline{I\left(\Sigma, \lambda_{0}\right)}$. Since $\overline{I^{-}\left(\lambda_{0}\right)}=\overline{I\left(\Sigma, \lambda_{0}\right)} \cup \overline{I^{-}\left(\lambda_{0}\right) \cap I^{-}(\Sigma)}$ and $S$ is neither in nor to the past of $\Sigma, S \nsubseteq \overline{I^{-}\left(\lambda_{0}\right)}$. Thus, for any compact $K \subset S, K \nsubseteq \overline{\mathrm{I}^{-}\left(\lambda_{0}\right)}$. However, $K \subset \mathrm{I}\left(\Sigma, \lambda_{0}+\epsilon\right)$ for all $\epsilon>0$, which means that every neighborhood of $\lambda_{0}$ contains a $p \in \mathrm{I}^{+}\left(\lambda_{0}\right)$ such that $K \subset \mathrm{I}^{-}(p)$, which means that $\mathrm{I}^{-}\left(\lambda_{0}\right)$ is not outer continuous. For $(M, g)$ globally hyperbolic, this is not possible.

Thus, $\mathrm{v}_{+}=\mathrm{v}\left(\lambda_{0}\right)=\mathrm{v}_{-}$, and hence $\mathrm{v}(\lambda)$ is a continuous function. The proof for the time-reversed case is identical.

Since $\mathrm{v}$ is a continuous, monotonically increasing function along $\xi(s)$ to the future of $\Sigma$, we can reparametrize the curves $\xi_{\lambda}(s)$ to $\xi_{\mathrm{v}}(s)$. $\xi_{\mathrm{v}}(s)$ then provides us with a one-parameter family of 
homeomorpisms between $\Sigma$ and $v=$ const hypersurfaces $\Sigma_{v}$ to its future. The space-time region $M_{\mathrm{v}} \equiv \overline{\mathrm{I}\left(\Sigma, \Sigma_{\mathrm{v}}\right)}$ can be regarded as a volume thickening of $\Sigma$ and is the desired continuum analog of a thickened antichain.

The continuum nerve simplicial complex analogous to $\mathcal{N}(\mathcal{A})$ may then be constructed from $M_{\mathrm{v}}$ as follows. $\Sigma_{\mathrm{v}}$ are the maximal or future-most elements of $M_{\mathrm{v}}$, and the past of any point $p$ $\in \Sigma_{\mathrm{v}}$ casts a shadow

$$
\sigma(p) \equiv \mathrm{I}^{-}(p) \cap \Sigma
$$

on $\Sigma$. The set $\mathcal{S}_{\infty} \equiv\left\{\sigma(p) \mid p \in \Sigma_{\mathrm{v}}\right\}$ is an open cover of $\Sigma$, since for every $s \in \Sigma$ there is a $p \in \Sigma_{\mathrm{V}}$ such that $s \in I^{-}(p)$. The associated nerve $\mathcal{N}\left(\mathcal{S}_{\infty}\right)$ is an infinite dimensional simplicial complex, arising from nonvanishing intersections of arbitrarily many sets in $\mathcal{S}_{\infty}$.

In the continuum limit in which the discreteness scale is taken to zero, $\mathcal{N}(\mathcal{A}) \rightarrow \mathcal{N}\left(\mathcal{S}_{\infty}\right)$. However, since our interest lies in the continuum approximation for which the discreteness scale is not taken to zero, it is more appropriate to use a locally finite subcover of $\mathcal{S}_{\infty}$. We do so by choosing a collection of points $\left\{m_{i}\right\}$ on $\Sigma_{\mathrm{v}}$ such that (a) the collection of shadows $\mathcal{S} \equiv\left\{\sigma\left(m_{i}\right)\right\}$ is a cover of $\Sigma$ and (b) any open set in $\Sigma_{v}$ contains only a finite number of $m_{i}$. Then $\mathcal{S}$ is a locally finite cover of $\Sigma$, and $\mathcal{N}(\mathcal{S})$ is the required continuum analog of $\mathcal{N}(\mathcal{A})$.

\section{A CONTINUUM RESULT}

Of course, one of the main questions we want to address is whether such a continuum nerve construction yields a simplicial complex which is homological to $\Sigma$ and hence to $M$. For example, in order to construct the Cěch cohomology, one starts with an ordered infinite collection $\left\{\mathcal{S}_{1}, \mathcal{S}_{2}, \ldots \mathcal{S}_{i}, \ldots\right\}$ of locally finite open coverings of $M$, such that $\mathcal{S}_{i+1}$ is a refinement of $\mathcal{S}_{i}$. The correct continuum cohomology is then recovered only in the limit $i \rightarrow \infty$, i.e., the limit of infinite refinement. This is reasonable in the continuum, since any finite refinement can contain sets that are "too large," with the result that the nerve simplicial complex masks nontrivial topological information.

However, for any fundamentally discrete theory like causal set theory, continuum structures smaller than the discreteness scale are unphysical. Hence, it should suffice to consider a finite cover. In particular, we wish to ask what topological information of $\Sigma$ is encoded in the nerve $\mathcal{N}(\mathcal{S})$ of the locally finite cover $\mathcal{S} \equiv\left\{\sigma\left(m_{i}\right)\right\}$ of $\Sigma$. More specifically, is $\mathcal{N}(\mathcal{S})$ homologous to $\Sigma$ ? A look at a simple example tells us that the answer in general is no.

Consider the $S^{1} \times \mathbb{R}$ cylinder space-time $\mathrm{d} s^{2}=-\mathrm{d} t^{2}+\mathrm{d} \theta^{2}$, with $\theta \in[0,2 \pi]$. If $\Sigma$ is the $t=0$ circle, then $\mathrm{V}= \pm t^{2}$ if $t<\pi$. For $t=3 \pi / 4$, let us construct a cover $\mathcal{S}_{1}$ as follows. The shadows from the pair of points $m_{1}=(3 \pi / 4,0)$ and $m_{2}=(3 \pi / 4, \pi)$ are sufficient to cover the $t=0$ slice and $\sigma\left(m_{1}\right) \cap \sigma\left(m_{2}\right)$ is the disjoint union of the two open intervals $(\pi / 4,3 \pi / 4) \sqcup(5 \pi / 4,7 \pi / 4)$ on the $t=0$ circle. $\mathcal{N}\left(\mathcal{S}_{1}\right)$ is then a single 1-simplex, which is not homological to $S^{1}$. On the other hand, for $t=\pi / 2$, a cover $\mathcal{S}_{2}$ is provided by the shadows from the three points $m_{1}=(\pi / 2,0), m_{2}$ $=(\pi / 2,2 \pi / 3)$, and $m_{3}=(\pi / 2,4 \pi / 3)$. Their intersections on the $t=0$ slice are the intervals $\sigma\left(m_{1}\right) \cap \sigma\left(m_{2}\right)=(\pi / 6, \pi / 2), \sigma\left(m_{2}\right) \cap \sigma\left(m_{3}\right)=(5 \pi / 6,7 \pi / 6)$, and $\sigma\left(m_{3}\right) \cap \sigma\left(m_{1}\right)=(3 \pi / 2,11 \pi / 6)$, respectively, from which we see that $\cap_{i=1}^{3} \sigma\left(m_{i}\right)=\emptyset . \mathcal{N}\left(\mathcal{S}_{2}\right)$ is then the boundary of a 2-simplex and therefore homological to $S^{1}$. This simple example shows us that the choice of cover $\mathcal{S}$ is crucial for determining whether $\mathcal{N}(\mathcal{S})$ has the correct homology.

A somewhat lesser known result in algebraic topology due to De Rham and Weil ${ }^{19}$ then gives us a criterion for $\mathcal{S}$ such that $\mathcal{N}(\mathcal{S})$ is not only homological but homotopic to $\Sigma$.

Theorem 1. (De Rham-Weil): The nerve of a convex cover of $\Sigma$ is homotopic to $\Sigma$.

Theorem 1 is also valid for a simple cover, whose elements are differentiably contractible as are all intersections (since our interest here is restricted to homology, it suffices that the intersections are acyclic, i.e., they contain no nontrivial cycles). From this we see why the two covers on the cylinder space-time give such different results. While the shadows are themselves differentiably contractible in the cover $\mathcal{S}_{1}$, their intersections are homotopic to two disconnected points, so that $\mathcal{S}_{1}$ is neither convex nor simple. For $\mathcal{S}_{2}$, the shadows and their intersections are both convex 
and contractible, resulting in a nerve which is homological to $\mathrm{S}^{1}$ in accordance with Theorem 1 .

The open cover of interest to us is the collection of shadow sets $\mathcal{S}$. As we have seen in the cylinder space-time, this is not always a convex cover. Nevertheless, as is apparent even for this simple space-time, for times $t \leqslant \pi / 2$, every shadow is, in fact, a convex set of the $t=0$ slice. This suggests that for $v$ below some critical value, one should always get shadows that are convex for any globally hyperbolic space-time. We will now show that this is indeed the case when the Cauchy surface $\Sigma$ is taken to be compact. We will take $(M, g)$ and $(\Sigma, h)$ to be at least $C^{2}$ differentiable.

Let $\mathfrak{r}$ be the convexity radius on the Cauchy slice $(\Sigma, h)$, where $h$ is the induced spatial metric. We again take $\xi^{a}$ to be a continuous, future-directed timelike vector field and $\xi(s)$ its integral curve which intersects $\Sigma$ at $s$. We use the volume function $v$ as defined in Eq. (2) to parametrize this curve and denote the shadows of the past sets of $\xi_{\mathrm{v}}(s)$ on $\Sigma$ by $\sigma_{s}(\mathrm{v})$ for $\mathrm{v}>0$. We also note that for $\Sigma$ compact, there exists a $\mathrm{v}_{\text {crit }}>0$ such that $\forall \mathrm{v} \geqslant \mathrm{v}_{\text {crit }}$, there exist $p \in \Sigma_{\mathrm{v}}$ such that $\sigma(p)=\Sigma$.

Now, any $s \in \Sigma$ is contained in a CNN N of $(M, g)$ such that $\mathrm{N} \cap \Sigma$ is also a CNN of $(\Sigma, h)$. We will term such an $\mathrm{N}$ a Cauchy-CNN or C-CNN. One way of constructing such a C-CNN is from a sufficiently small CNN $\mathrm{N}^{\prime} \ni s$ of $(\Sigma, h)$, such that its domain of dependence $D\left(\mathrm{~N}^{\prime}\right)$ is itself a CNN of $(M, g)$.

Lemma 2: For every $s \in \Sigma$ and $\xi^{a}$ a future-directed timelike vector field, $\exists a \mathrm{v}_{s}, \mathrm{v}_{\text {crit }}>\mathrm{v}_{s}$ $>0$, such that for any $0<\mathrm{v}<\mathrm{v}_{s}$,

1. $\operatorname{diam}\left(\overline{\sigma_{s}(\mathrm{v})}\right)<\mathfrak{r}$, the convexity radius of $\Sigma$, and

2. $\overline{\partial \sigma_{s}(\mathrm{v})}$ has positive principal curvatures with respect to its outward normal for space-time dimension $\mathfrak{n} \geqslant 3$.

Proof: We first show that for any $\mathrm{v}_{1}, \mathrm{v}_{2}, \mathrm{v}_{\text {crit }}>\mathrm{v}_{1}>\mathrm{v}_{2}>0, \sigma_{s}\left(\mathrm{v}_{2}\right) \subset \sigma_{s}\left(\mathrm{v}_{1}\right)$. By transitivity, $\overline{\sigma_{s}\left(\mathrm{v}_{2}\right)} \subseteq \overline{\sigma_{s}\left(\mathrm{v}_{1}\right)}$. The strict inclusion is proven as follows. Assume that $\sigma_{s}\left(\mathrm{v}_{2}\right)=\sigma_{s}\left(\mathrm{v}_{1}\right)$ for $\mathrm{v}_{2}<\mathrm{v}_{1}$. Any $x \in \partial \overline{\sigma_{s}\left(\mathrm{v}_{2}\right)}$ lies on $\overline{\partial\left(\mathrm{I}^{-}\left(\mathrm{v}_{2}\right)\right)}$ which by global hyperbolicity means that $x \rightarrow \xi_{\mathrm{v}_{2}}(s)$, i.e., they are null related. Since $\xi_{\mathrm{v}_{2}}(s)<<\xi_{\mathrm{v}_{1}}(s)$, this means that $x<<\xi_{\mathrm{v}_{1}}(s)$ or $x$ lies in $\mathrm{I}^{-}\left(\xi_{\mathrm{v}_{1}}(s)\right)$. ${ }^{15}$ But $x \in \partial \overline{\left(\sigma_{s}\left(\mathrm{v}_{2}\right)\right)} \subset \partial \overline{\left(\mathrm{I}^{-}\left(\mathrm{v}_{1}\right)\right)}$ as well, which is a contradiction. Therefore, $\overline{\partial \sigma_{s}\left(\mathrm{v}_{2}\right)} \cap \overline{\partial \sigma_{s}\left(\mathrm{v}_{1}\right)}=\emptyset$ and hence $\overline{\sigma_{s}\left(\mathrm{v}_{2}\right)} \subset \overline{\sigma_{s}\left(\mathrm{v}_{1}\right)}$, which implies that $\sigma_{s}\left(\mathrm{v}_{2}\right) \subset \sigma_{s}\left(\mathrm{v}_{1}\right)$, since $\sigma_{s}(\mathrm{v})$ is an open set. Thus, the $\sigma_{s}\left(\mathrm{v}_{i}\right)$ are nested one inside the other.

Now, for every CNN $\mathrm{N}^{\prime} \ni s$, in $(\Sigma, h)$, there exists a $\mathrm{v}_{s}^{\prime}>0$ such that $\sigma_{s}(\mathrm{v}) \subset \mathrm{N}^{\prime}$, for all $\mathrm{V}_{s}^{\prime}>\mathrm{v}>0$. Let $D^{+}\left(\overline{\mathrm{N}^{\prime}}\right)$ be the future domain of dependence of $\overline{\mathrm{N}^{\prime}}$ (a closed achronal set) and $H^{+}\left(\overline{\mathrm{N}^{\prime}}\right)$ its future boundary. Let $p \in \xi_{\mathrm{v}}(s)$ such that $s<<p<<r$, where $r \in \xi_{\mathrm{v}}(s) \cap H^{+}\left(\overline{\mathrm{N}^{\prime}}\right) \neq \emptyset$. Then, $\mathrm{I}^{-}(p) \cap \Sigma=\sigma_{s}(\mathrm{v}(p)) \subset \overline{\mathrm{N}}^{\prime}$ since $p \in D^{+}\left(\overline{\mathrm{N}^{\prime}}\right)$, so that $\overline{\sigma_{s}(\mathrm{v}(p))} \subseteq \overline{\mathrm{N}}^{\prime}$. Assume equality. Then for all $x \in \partial \overline{\sigma_{s}(\mathrm{v}(p))}=\partial \overline{\mathrm{N}^{\prime}}, x \rightarrow p<<r$, or $x<<r$. But since $r \in H^{+}\left(\overline{\mathrm{N}^{\prime}}\right)$, by globally hyperbolicity, there exists an $x^{\prime} \in \partial \overline{\mathrm{N}^{\prime}}$ for which $x^{\prime} \rightarrow r$, which implies a contradiction. Hence $\sigma_{s}(\mathrm{v}(p)) \subset \overline{\mathrm{N}^{\prime}}$. Thus, for all $\mathrm{v}>\mathrm{v}_{s}^{\prime} \equiv \mathrm{v}(r), \sigma_{s}(\mathrm{v}) \subset \mathrm{N}^{\prime}$.

Moreover, let $\mathrm{N}^{\prime}$ such that $\operatorname{diam}\left(\overline{\mathrm{N}^{\prime}}\right)<\mathfrak{r}$. Again, $\mathrm{v}_{s}^{\prime}>0$ is such that for all $0<\mathrm{v}<\mathrm{v}_{s}^{\prime}$, $\sigma_{s}(\mathrm{v}) \subset \mathrm{N}^{\prime}$. Let $p, q \in \overline{\partial \sigma_{s}(\mathrm{v})}$ such that $d(p, q)=\operatorname{diam}\left(\overline{\sigma_{s}(\mathrm{v})}\right)$ which is the length of the (unique) geodesic $\gamma$ in $\mathrm{N}^{\prime}$ from $p$ to $q$. Let $\gamma(0) \equiv p, \gamma(1) \equiv q$, and $\gamma(1+\epsilon) \equiv r$. For small enough $\epsilon>0$, $r \in N^{\prime} . d(p, r)$ is therefore the arclength of $\gamma$ from $p$ to $r$, so that $d(p, r)=d(p, q)+d(q, r)$, thus implying that $\mathfrak{r}>\operatorname{diam}\left(\overline{\mathrm{N}^{\prime}}\right) \geqslant d(p, r)>\operatorname{diam}\left(\overline{\sigma_{s}(\mathrm{v})}\right)$. This proves point 1 .

We can do better, i.e., find the largest possible $\mathrm{v}_{s}^{\prime}$ for which point 1 is true by taking the supremum over all such C-CNN's $\mathrm{N}^{\prime}$, i.e., $\mathrm{v}_{s}^{\prime \sup } \equiv \sup _{\mathrm{N}^{\prime}} \mathrm{v}_{s}^{\prime}>0$.

To prove point 2 we note that for a flat (i.e., zero extrinsic curvature) Cauchy hypersurface in Minkowski space-time, the principal curvatures of the boundary of the shadows of a past light cone on it are strictly positive. We show that we can construct "small enough" neighborhoods of $s$ such that the deviation from flatness is sufficiently small for this to be possible.

Since the boundaries of the shadows $\overline{\partial\left(\sigma_{s}(\mathrm{v})\right)}$ do not intersect for different $\mathrm{v}<\mathrm{v}_{\text {crit }}$ by outer continuity, the $n-2$ dimensional surfaces $\partial \overline{\sigma_{s}(\mathrm{v})} \simeq \mathrm{S}^{n-2}$ provide a foliation of $\Sigma$ centered at $s$. The boundary of $\mathrm{J}^{-}\left(\xi_{\mathrm{v}}(s)\right)$ is generated by past directed null geodesics which intersect $\Sigma$ at $\overline{\partial \sigma_{s}(\mathrm{v})}$. Let y coordinatize these $n-2$ null directions, i.e., the $S^{n-2}$. In $2+1$-dimensions, for example, $\mathbf{y}=\theta$ the 
coordinate on a circle, $\mathrm{S}^{1}$. Let $\mathfrak{F}$ be the set of monotonically increasing functions of $\mathrm{v}$ which vanish at $\mathrm{v}=0$. Since the deviation from flatness decreases with $\mathrm{v}$, a given function in $\mathfrak{F}$ can serve to parametrize the deviation from flatness of the metric in a CNN of $s$ in $(\Sigma, h)$ along any given null direction $\mathbf{y}$ projected onto $\Sigma$.

The set $(\mathrm{v}, \mathbf{y})$ are therefore Riemann normal coordinates on a $\mathrm{CCN} \mathrm{N}^{\prime}$ of $(\Sigma, h)$, centered at $s$ and the induced spatial metric is $h_{a b}(\mathrm{v}, \mathbf{y})=\delta_{a b}+\mathcal{O}(f), f(\mathrm{v}) \in \mathfrak{F}$, where the $\mathbf{y}$ dependence on the right hand side is absorbed into the second term. Let $n^{a}$ be the unit normal to $\mathrm{N}^{\prime}$ in $(M, g)$, so that the extrinsic curvature of $\mathrm{N}^{\prime}$ is $K_{a b}=\frac{1}{2} \mathfrak{L}_{n} h_{a b}$. Choose $\mathrm{N}^{\prime}$ to be small enough so that $n^{a}$ is nearly constant over $\mathrm{N}^{\prime}$, i.e., $n^{a}(\mathbf{v}, \mathbf{y})=n^{a}(s)+\mathcal{O}\left(f^{\prime}\right)$ with $f^{\prime}(\mathbf{v}) \in \mathfrak{F}$, again with the $\mathbf{y}$ dependence absorbed into the second term. Since $\Sigma$ is smoothly embedded into $(M, g), n^{a}$ and hence $K_{a b}$ vary smoothly in $(M, g)$. Christoffels $\Gamma_{a b}^{c}(\mathrm{v}, \mathbf{y})=\mathcal{O}(f)$ and vanish at $s$, so that extrinsic curvature $K_{a b}$ $=\mathcal{O}(\widetilde{f})$, where $\tilde{f}$ is the more dominating of the two functions $f, f^{\prime}$, as $\mathbf{v} \rightarrow 0$.

If $l_{a b}(\mathrm{v}, \mathbf{y})$ is the induced metric on $\overline{\partial \sigma_{s}(\mathrm{v})}$ and $m^{a}(\mathrm{v})$ its outward normal, the foliation by $\partial \overline{\sigma_{s}(\mathrm{v})}$ allows us to express $h_{a b}(\mathrm{v})=l_{a b}(\mathrm{v}, \mathbf{y})+m_{a}(\mathrm{v}, \mathbf{y}) m_{b}(\mathrm{v}, \mathbf{y})$. This means that $l_{a b}(\mathrm{v}, \mathbf{y})$ and $m^{a}(\mathbf{v}, \mathbf{y})$ each differ from their flat space counterparts, i.e., on $\left(\mathbf{N}^{\prime}, \delta\right)$, by at most some $\mathcal{O}\left(f_{m}\right)$, $f_{m} \in \mathfrak{F}$, where $f_{m}$ can differ from $f$ if it is more dominating as $\mathbf{v} \rightarrow 0$. Thus, $l_{a b}(\mathrm{v}, \mathbf{y})=l_{a b}^{\mathrm{F}}(\mathbf{v}, \mathbf{y})$ $+\mathcal{O}\left(f_{m}\right)$ and $m_{a}(\mathbf{v}, \mathbf{y})=m_{a}^{\mathrm{F}}(\mathbf{v}, \mathbf{y})+\mathcal{O}\left(f_{m}\right)$, where the $\mathrm{F}$ labels the quantities with respect to the flat metric $\delta_{a b}$ on $\mathrm{N}^{\prime}$. The principal curvatures of $\overline{\partial \sigma_{s}(\mathrm{v})}$ are therefore $\kappa_{i}(\mathrm{v}, \mathbf{y})=\kappa_{i}^{\mathrm{F}}(\mathrm{v}, \mathbf{y})+\mathcal{O}\left(f_{m}\right), i$ $=1, \ldots, n-2$, with $\kappa_{i}^{\mathrm{F}}(\mathrm{v}, \mathbf{y})$ the principal curvature of these surfaces with respect to $\delta_{a b}$ on $\mathrm{N}^{\prime}$. For example, for a spherically symmetric foliation about $s$ in a flat metric, the $\kappa_{i}^{\mathrm{F}}(\mathrm{v})$ are positive and diverge to $+\infty$ as $\mathbf{v} \rightarrow 0$.

In calculating the dominant contributions to the principal curvatures $\kappa_{i}$ we have not yet made any assumptions about the foliation $\left\{\partial \overline{\left(\sigma_{s}(\mathrm{v})\right)}\right\}$ which depends on the space-time metric. Without this further input, the $\kappa_{i}^{\mathrm{F}}$ will not in general be positive.

Let us therefore choose a C-CNN N $\ni s$ in $(M, g)$ such that not only does $\mathrm{N}^{\prime} \equiv \mathrm{N} \cap \Sigma$ satisfy the above conditions but the space-time metric $g_{a b}=\eta_{a b}+\mathcal{O}\left(f^{\prime \prime}\right)$ in $\mathrm{N}$ with $f^{\prime \prime}(\mathrm{v}) \in \mathfrak{F}$. For example, for $\mathrm{N}$ strictly Minkowskian, and $\mathrm{N}^{\prime}$ with zero extrinsic curvature, the $\overline{\partial \sigma\left(\mathrm{v}_{F}\right)}$ have strictly positive $\kappa_{i}^{\mathrm{F}}$, where $\mathrm{v}_{F}$ represents the equivalent volume in Minkowski space-time. Thus, the volume $\mathrm{v}$ $=\mathrm{v}_{F}+\mathcal{O}\left(f^{\prime \prime}\right)$, and the principal curvatures of $\overline{\partial \sigma_{s}(\mathrm{v})}, \kappa_{i}(\mathrm{v}, \mathbf{y})=\kappa_{i}^{\mathrm{F}}\left(\mathrm{v}_{F}, \mathbf{y}\right)+\mathcal{O}(\tilde{f})$, where $\tilde{f}$ is now the more dominating of the functions $f_{m}, f^{\prime}, f^{\prime \prime}$ as $\vee \rightarrow 0$. We therefore see that for any fixed null direction $\mathbf{y}$ there exists a $\mathbf{v}(\mathbf{y})>0$ such that for all $0<\mathbf{v}<\mathbf{v}(\mathbf{y}), \kappa_{i}\left(\mathbf{v}, \mathbf{y}^{a}\right)>0$. Since $\Sigma$ is a smooth Cauchy hypersurface, the boundaries of the shadows are smooth in a C-CNN. Thus, $\kappa_{i}(v, \mathbf{y})$ is continuous with respect to $\mathbf{y}$, i.e., over the set of null directions $S^{n-2}$. If $\mathrm{v}<\mathrm{v}(\mathbf{y})$ for some $\mathbf{y}$ $\in \mathbb{S}^{n-2}$, then since $\kappa_{i}(\mathrm{v}, \mathbf{y})>0$ there exists a neighborhood $U$ of $\mathbf{y}$ in $S^{n-2}$ such that for all $\mathbf{y}^{\prime}$ $\in U, \kappa_{i}\left(\mathrm{v}, \mathbf{y}^{\prime}\right)>0$. Thus, $\mathrm{v}\left(\mathbf{y}^{\prime}\right) \geqslant \mathrm{v}(\mathbf{y})$. Now, for every $\mathbf{y}, \mathrm{v}(\mathbf{y})>0$. However, if $\inf _{\mathrm{y}} \mathrm{v}(\mathbf{y})=0$, then there is an infinite sequence $\left\{\mathrm{v}\left(\mathbf{y}_{i}\right)\right\} \rightarrow 0$. The corresponding sequence in $\mathbb{S}^{n-2},\left\{\mathbf{y}_{i}\right\} \rightarrow \mathbf{y}_{0} \in \mathbb{S}^{n-2}$. Thus, for every neighborhood $U$ of $\mathbf{y}_{0}$ there exists a $j$ such that for all $i>j, \mathrm{v}\left(\mathbf{y}_{i}\right)<\mathbf{v}\left(\mathbf{y}_{0}\right)$ and $\mathrm{v}\left(\mathbf{y}_{0}\right)>0$. This is a contradiction. Hence, $\mathrm{v}_{s}^{\prime \prime}=\inf _{\mathbf{y}} \mathrm{v}(\mathbf{y})>0$, which proves point 2 .

We may now obtain the largest possible $\mathrm{v}_{s}^{\prime \prime}$ by now varying over all $\mathrm{N}^{\prime}$ which satisfy the above criteria to obtain $\mathrm{v}_{s}^{\prime \prime \text { sup }}=\sup _{\mathrm{N}^{\prime}} \mathrm{v}_{s}^{\prime \prime}>0$.

Thus, for every $s \in \Sigma$, there exists a $\mathrm{v}_{s}=\min \left(\mathrm{v}_{s}^{\prime \text { sup }}, \mathrm{v}_{s}^{\prime \prime s u p}\right)>0$ for which 1 and 2 are satisfied.

For a fixed timelike vector field $\xi^{a}$, although $\mathrm{v}_{s}>0$ for each $s \in \Sigma$, we now show that $\inf _{s} \mathrm{v}_{s}$ $>0$ using global hyperbolicity and the compactness of the Cauchy hypersurfaces $\Sigma$. Let $\gamma(\alpha)$ be a continuous curve in $(\Sigma, h)$ through $s \in \Sigma$, with $\gamma(0)=s$. The homeomorphism $\xi_{v}: \Sigma \rightarrow \Sigma_{v}$ takes $\gamma(\alpha) \rightarrow \gamma_{v}(\alpha) \equiv \xi_{v} \circ \gamma(\alpha)$. Let $S(\alpha) \equiv \overline{\sigma_{\gamma(\alpha)}(v)}$, i.e., the closure of the shadows of past light cones of points in $\gamma_{v}(\alpha)$ onto $\Sigma$. $\{\partial S(\alpha)\}$ is then a family of $S^{n-2}$ s. We now prove a couple of claims.

Claim 1: The family $\{S(\alpha)\}$ is continuous with respect to $\alpha$.

Proof: Let $f(\alpha, \mathbf{v}, \mathbf{y})=0$ be the functions defining the $\partial S(\alpha)$ 's. Assume that $f$ is not continuous at $s$ or $\alpha=0$, so that $\lim _{\alpha \rightarrow 0} f(\alpha, \mathrm{v}, \mathbf{y})=\tilde{f}(0, \mathrm{v}, \mathbf{y}) \neq f(0, \mathrm{v}, \mathbf{y})$. Equivalently, $\lim _{\alpha \rightarrow 0} S(\alpha)=\tilde{S}(0)$ $\neq S(0)$. Let $\left\{s_{i}\right\}$ be an infinite sequence of points on $\gamma(\alpha)$ which converge to $s$, with $s_{i} \equiv \gamma\left(\alpha_{i}\right)$ and $0<\alpha_{i+1}<\alpha_{i}$. By definition $\widetilde{S}(0)$ is the limit set of $\left\{S\left(\alpha_{i}\right)\right\}$, i.e., for every $q \in \widetilde{S}(0)$ and any open 
$O \ni q$ there exists a $j$ such that for all $i>j, O \cap S\left(\alpha_{i}\right) \neq \emptyset$. Let $\left\{p_{i}\right\}$ be the corresponding sequence on $\gamma_{\mathrm{v}}(\alpha)$, i.e., $p_{i} \equiv \xi_{\mathrm{v}}\left(s_{i}\right)$, which converges to $p \equiv \gamma_{v}(0)$.

Now, let $q \in \tilde{S}(0) \backslash S(0)$, which is nonempty by definition. Since $S(0)$ is closed, there exists an open $O \ni q$ such that $O \cap S(0)=\emptyset$. Any compact subset $K$ of $O$ therefore lies in $S(0)^{c}$ and hence in $\frac{I^{-}(p)^{c}}{\text {. Let }} q \in K \subset O$ and let $q \in O^{\prime} \subset K$ be open. For any such $q, O^{\prime}$, then, there exists a $j$ such that $\forall i>j, O^{\prime} \cap S\left(\alpha_{i}\right) \neq \emptyset$ since $q \in \tilde{S}(0)$. Therefore $K \cap S\left(\alpha_{i}\right) \neq \emptyset$ and $K \cap \overline{\mathbf{I}^{-}\left(p_{i}\right)} \neq \emptyset$. Thus, every neighborhood of $p$ contains a $p_{i}$ such that $K \cap \overline{I^{-}\left(p_{i}\right)^{c}} \neq \emptyset$. This contradicts causal continuity.

Claim 2: If $\mathrm{v}<\mathrm{v}_{s}$ then $\exists \delta>0$ such that $\forall 0<\alpha<\delta, S(\alpha)$ satisfies points 1 and 2 of Lemma 2.

Proof: Continuity of the $S(\alpha)$ with respect to $\alpha$ means continuity of its associated geometric properties. Since $S(\alpha)$ are continuous, there exists a homeomorphism $\varphi_{\alpha}: S(0) \rightarrow S(\alpha)$. Let $\chi(0, t)$ be a (continuous) segment from $x(0)$ to $y(0)$ on $S(0)$ mapped by $\varphi_{\alpha}$ to $\chi(\alpha, t)$ with end points $x(\alpha), y(\alpha) \in S(\alpha)$. The length $\ell(\chi(\alpha, t))$ is itself a continuous function of $\alpha$ because of the continuity of the metric $h_{a b}$ on $\Sigma$. Hence, since $\ell(\chi(0, t))<\mathfrak{r}$, this means that there exists a $\delta>0$ such that for all $0<\alpha<\delta, \ell(\chi(\alpha, t))<\mathfrak{r}$. Therefore $d(x(\alpha), y(\alpha))<\mathfrak{r}$. Again, continuity of $h_{a b}$ and the $\partial S(\alpha)$ means that the principal curvatures $\kappa_{i}(\alpha)$ are continuous with respect to $\alpha$. Since $\kappa_{i}(0)$ $>0$, there exists a $\delta^{\prime}>0$ such that $\forall 0<\alpha<\delta^{\prime}, \kappa_{i}(\alpha)>0$ for all $i$.

Thus, there exists a $\delta^{\prime \prime}=\min \left(\delta, \delta^{\prime}\right)>0$ such that $\forall 0<\alpha<\delta^{\prime \prime}$ points 1 and 2 of Lemma 2 are satisfied.

Thus, for every $s \in \Sigma$, there exists a neighborhood $O$ of $s$ such that for all $s^{\prime} \in O, \mathrm{v}_{s} \leqslant \mathrm{v}_{s^{\prime}}$. Since $\Sigma$ is compact, an argument similar to the one used to show $\mathrm{v}_{s}^{\prime \prime}>0$ in the proof of Lemma 2 can be used to show that $\widetilde{\mathrm{V}}^{\prime} \equiv \inf _{s \in \Sigma} \mathbf{V}_{s}>0$.

By varying over the timelike vector fields $\xi^{a}$, we obtain a convexity volume of $\Sigma$ which is the largest $\mathbf{v}^{\prime}$ for a given $(\Sigma, h) \subset(M, g)$

$$
\widetilde{\nabla} \equiv \sup _{\xi^{a}} \widetilde{\nabla}^{\prime}, \quad 0<\widetilde{\nabla} \leqslant \mathrm{v}_{\text {crit }} .
$$

The positivity of the principal curvatures of the boundaries of the shadows then implies, by the maximum principle, ${ }^{17}$ that for any $p, q \in \sigma_{s}(\mathrm{v})$ and $0<\mathrm{V}<\widetilde{\mathrm{v}}$, if there exists a geodesic from $p$ to $q$ of arclength $<\operatorname{diam}\left(\sigma_{s}(\mathrm{v})\right)$, then it is unique and lies in $\sigma_{s}(\mathrm{v})$ for $\mathfrak{n} \geqslant 3$. For $\mathfrak{n}=2$, we do not require 2 since as long as 1 is satisfied, any geodesic from $p$ to $q$ in $\sigma_{s}(\mathrm{v})$ of arclength $<\operatorname{diam}\left(\sigma_{s}(\mathrm{v})\right)$ must lie in $\sigma_{s}(\mathrm{v})$. Hence, $\sigma_{s}(\mathrm{v})$ is convex with respect to $\mathfrak{r}$ for all $\mathfrak{n} \geqslant 2$.

Thus, the collection $\mathcal{S}_{\mathrm{v}}^{\infty} \equiv\left\{\sigma_{s}(\mathrm{v})\right\}$ for any $\mathrm{V}<\widetilde{\mathrm{V}}$ forms an open covering of $\Sigma$ whose elements are convex sets with respect to $\mathfrak{r}$. A locally finite cover $\mathcal{S}_{V}$ can be obtained from $\mathcal{S}_{v}^{\infty}$ as follows. Since $\Sigma$ is paracompact, $\mathcal{S}_{v}^{\infty}$ admits a locally finite refinement, i.e., a locally finite cover $\mathcal{V}$ of $\Sigma$ such that for every $V_{i} \in \mathcal{V}$, there exists a $\sigma_{s}(\mathrm{v}) \in \mathcal{S}_{\mathrm{v}}^{\infty}$ such that $V_{i} \subset \sigma_{s}(\mathrm{v})$. For every $V_{i}$, choose an $s_{i}$ such that $V_{j} \subset \sigma_{s_{i}}(\mathrm{~V})$ implies $j=i$. Since $\mathcal{V}$ is a cover of $\Sigma$, so is $\mathcal{S}_{\mathrm{V}} \equiv\left\{\sigma_{s_{i}}(\mathrm{~V})\right\}$. Local finiteness of $\mathcal{V}$ implies that every $p \in \Sigma$ has a neighborhood $\mathrm{N} \ni p$ which intersects only a finite number of $V_{i}$. Since each $V_{i}$ is contained in a unique $\sigma_{s_{i}}(\mathrm{v}), \mathrm{N}$ also intersects only a finite number of $\sigma_{s_{i}}(\mathrm{v})$, so that $\mathcal{S}_{\mathrm{V}}$ is a locally finite convex cover of $\Sigma$. $\mathcal{S}_{\mathrm{v}}$ is therefore a convex open cover of $\Sigma$ and hence, using Theorem 1 we have shown the following.

Theorem 2: The nerve $\mathcal{N}\left(\mathcal{S}_{\mathrm{V}}\right)$ is homotopic to $\Sigma$ and thence to $M$.

In order to make the continuum-discrete comparison, which is the main goal of this paper, one would like to obtain the discrete analog of the cover $\mathcal{S}_{\mathrm{v}}$. While it is tempting to use the obvious identification with the collection $\mathcal{A}$ as suggested at the end of Sec. III, one must proceed with caution. Since the continuum-discrete correspondence for causets maps space-time volume (and not spatial volume) to cardinality, we need to find a collection of open sets in space-time corresponding to $\mathcal{S}_{\mathrm{v}}$. The natural choice is the collection of past sets, $\mathcal{I}_{\mathrm{v}} \equiv\left\{\mathrm{I}_{i} \equiv \mathrm{I}\left(\Sigma, \xi_{\mathrm{v}}\left(s_{i}\right)\right)\right\}$. For ease of notation, let $\xi_{i} \equiv \xi\left(s_{i}\right), m_{i}=\xi_{\mathrm{v}}\left(s_{i}\right)$, and $\sigma_{i} \equiv \sigma_{\mathrm{v}}\left(s_{i}\right)$. Since $\sigma_{i}=\mathrm{I}^{-}\left(m_{i}\right) \cap \Sigma$, the map $\Psi: \mathcal{I}_{\mathrm{v}} \rightarrow \mathcal{S}_{\mathrm{v}}$ is onto. If $\Psi$ were in addition one-to-one, then $\sigma_{i}=\sigma_{j} \Rightarrow i=j$. Assume otherwise, i.e., $\sigma_{i}=\sigma_{j}, i \neq j$, so that $\partial \bar{\sigma}_{i}=\partial \bar{\sigma}_{j}$. Since the space-time is globally hyperbolic $\forall x \in \partial \bar{\sigma}_{i}, x \rightarrow m_{i}$ and $x \rightarrow m_{j}$. Now, $s_{i}, s_{j} \in \mathrm{I}^{-}\left(m_{i}\right) \cap \mathrm{I}^{-}\left(m_{j}\right)$, which means that $\xi_{j} \cap\left(\mathrm{I}\left(\Sigma, m_{i}\right) \cap \mathrm{I}\left(\Sigma, m_{j}\right)\right) \neq \emptyset$. Since $m_{i}$ and $m_{j}$ are spacelike to each other, $\xi_{j}$ must leave $\partial \overline{\left(\Sigma, m_{i}\right)}$ at some $y=\xi_{j} \cap \partial \overline{I^{-}\left(m_{i}\right)}$. Therefore, $y \rightarrow m_{i}$ and $y$ 
$<<m_{j}$. Since $y \in \partial \overline{I^{-}\left(m_{i}\right)}$, there exists $x \in \partial \overline{\sigma_{i}}$ such that $x \rightarrow y \rightarrow m_{i}$. However, since $x \rightarrow y<<m_{j}$, this means that $x<<m_{j},{ }^{15}$ which is a contradiction. Hence $\Psi$ is one-to-one.

Consider the nonempty intersection $\sigma_{i_{1} \ldots i_{k}} \equiv \sigma_{i_{1}} \cap \cdots \cap \sigma_{i_{k}} \neq \emptyset$, which lies in $\mathrm{I}_{i_{1} \ldots i_{k}}^{-}$ $\equiv \mathrm{I}^{-}\left(p_{i_{1}}\right) \cap \cdots \cap \mathrm{I}^{-}\left(p_{i_{k}}\right)$. Let $a \in \sigma_{i_{1} \ldots i_{k}}$ and $\mathrm{N} \ni a$ such that $\mathrm{N} \subset \mathrm{I}_{i_{1} \ldots i_{k}}^{-}$. Then, $\mathrm{I}^{+}(\Sigma) \cap \mathrm{N} \subset \mathrm{I}_{i_{1} \ldots i_{k}}$ $\equiv \mathrm{I}_{i_{1} \ldots i_{k_{*}}}^{-} \cap \mathrm{I}^{+}(\Sigma)$ implies that $\mathrm{I}_{i_{1} \ldots i_{k}} \neq \emptyset$. Thus every $k$-simplex in $\mathcal{N}(\mathcal{S})$ maps to a $k$-simplex in $\mathcal{N}(\mathcal{I})$, i.e., $\Psi^{*}: \mathcal{N}(\mathcal{I}) \rightarrow \mathcal{N}(\mathcal{S})$ is onto. Since $\mathrm{I}_{i_{1} \ldots i_{k}} \subset \mathrm{I}^{+}(\Sigma)$, for every $y \in \mathrm{I}_{i_{1} \ldots i_{k}} \neq \emptyset$, there exists an $a \in \Sigma$ such that $a<<y$ and hence $a \in \mathrm{I}_{i_{1} \ldots i_{k}}^{-} \cap \Sigma=\sigma_{i_{1} \ldots i_{k}} \neq \emptyset$. Thus $\left(\Psi^{*}\right)^{-1}: \mathcal{N}\left(\mathcal{S}_{\mathrm{v}}\right) \rightarrow \mathcal{N}\left(\mathcal{I}_{\mathrm{v}}\right)$ is onto and hence $\Psi^{*}$ is a bijection. From Theorem 2, we see the following.

Corollary 1: $\mathcal{N}\left(\mathcal{I}_{\mathrm{v}}\right)$ is homotopic to $\Sigma$ and thence to $M$.

The cover $\mathcal{S}_{\mathrm{v}}$ of $\Sigma$ obtained from the causal structure can therefore be used to obtain a discretization of $\Sigma$, which is homotopic to $\Sigma$. This provides a new way of obtaining a faithful finitary topology from $\Sigma^{12}$ using the geometry of the space-time. It is tempting at this stage to speculate on how geometrical information may be extracted from the finitary topology. Namely, starting from a purely topological discretization of $\Sigma$ using a simple open covering, if one simply assumes this to be a convex cover $\mathcal{S}_{\mathrm{v}}$ for some choice of $\mathrm{v}$, then at least partial construction of a space-time geometry may be possible. However, we leave such investigations aside for now.

It is important to understand the role played by the convexity volume $\widetilde{v}$ associated with every $\Sigma$. For any $\mathrm{v}>\widetilde{\mathrm{v}}$, there exist sets $\sigma_{i} \in \mathcal{S}_{\mathrm{v}}$ which do not satisfy 1 and 2 of Lemma 2 and hence are not convex. However, while Theorem 1 gives a sufficient condition for $\mathcal{N}\left(\mathcal{S}_{\mathrm{V}}\right)$ to be homotopic to $\Sigma$, it is not a necessary condition. In other words, it is possible that there exist $\mathbf{v}>\widetilde{v}$ such that $\mathcal{N}\left(\mathcal{S}_{\mathrm{v}}\right)$ is homologically equivalent to $\Sigma$, even though $\mathcal{S}_{\mathrm{v}}$ is not a convex cover, as long as it is a simple cover. Thus, at best, $\widetilde{v}$ is a lower bound for which Theorem 2 is valid.

For the purposes of the next section it will be useful to find a slight generalization of $\mathcal{I}_{\mathrm{v}}$ and $\mathcal{S}_{\mathrm{v}}$ to accommodate sets associated with different v's. Let $\mathrm{M} \equiv\left\{m_{i}=\xi_{v_{i}}\left(s_{i}\right)\right\}$ be a collection of spacelike related points, and $\mathcal{I} \equiv\left\{\mathrm{I}_{i}^{\prime} \equiv \mathrm{I}\left(\Sigma, m_{i}\right)\right\}, \mathcal{S} \equiv\left\{\sigma_{\mathrm{v}_{i}}\left(s_{i}\right) \equiv \mathrm{I}^{-}\left(\xi_{\mathrm{v}_{i}}\left(s_{i}\right) \cap \Sigma\right)\right\}$, for $\mathrm{v}_{i}<\widetilde{\mathrm{v}}, \forall i$. Each of the $\sigma_{\mathrm{v}_{i}}\left(s_{i}\right)$ is therefore convex. Further, if the $\mathrm{M}$ are chosen such that $\mathcal{S}$ is a cover of $\Sigma$, then Theorem 2 and Corollary 1 imply the following.

Corollary 2: $\mathcal{N}(\mathcal{S})$ and $\mathcal{N}(\mathcal{I})$ are homotopic to $\Sigma$ and thence to $M$.

We end this section with some qualitative comments on the above foliation $\left\{\Sigma_{v}\right\} \times \mathbb{R}$ of $M$. Consider the sandwiched region $M_{\mathrm{v}}$, with boundaries $\Sigma$ and $\Sigma_{\mathrm{v}}$, for either $\mathrm{v}>0$ or $\mathrm{v}<0$. The volume thickening has the effect of "smoothing out" $\Sigma$, so that $\Sigma_{v}$ has a more "uniformized" or homogenized extrinsic curvature compared to $\Sigma$, both to the past and the future of $\Sigma$. This is a "dissipative" process analogous to a heat equation and is therefore irreversible in general: the volume foliation of $M$ with respect to some $\Sigma_{\mathrm{v}_{0}}, \mathrm{v}_{0}>0$ is not in general equivalent to that with respect to $\Sigma$, and the leaves of the foliation coincide only at $\Sigma_{v_{0}}$, so that a past volume thickening of $\Sigma_{\mathrm{v}_{0}}$ will in general not contain $\Sigma$ as a slice. Such uniformizing foliations may find an application in studies of Lorentzian geometry, analogous to the Ricci flows in Riemannian geometry. ${ }^{20}$

\section{THE CORRESPONDENCE}

We start with a causal set $C$ that faithfully embeds into a globally hyperbolic space-time $(M, g)$ at density $V_{c}^{-1}$,

$$
\Phi: C \rightarrow(M, g) .
$$

$V_{c}$ is the cutoff volume which sets the discreteness scale near which the manifold approximation of the causal set $C$ breaks down. is

The probability of finding $n$ points in a given volume $V$ for a Poisson sprinkling at density $V_{c}^{-1}$ 


$$
P_{n}(V)=\frac{1}{n !} \exp ^{-V / V_{c}} \times\left(\frac{V}{V_{c}}\right)^{n}
$$

which implies that $\bar{n}=V / V_{c}$, with standard deviation $\sigma=\sqrt{V / V_{c}}$. The relative width $\sigma / \bar{n}$ of this distribution decreases with increasing $\bar{n}$. Hence, for $V \gg V_{c}$ not only is $P_{n}(V)$ appreciable only for $n \gg 1$ but $n=\mathcal{O}\left(V / V_{c}\right)$ with probability close to 1 . This feature of the Poisson distribution will help guide us through the main results of this section.

In Sec. III we constructed the nerve $\mathcal{N}(\mathcal{P})$ associated with a thickened antichain $\mathrm{T}_{\mathrm{n}}(A)$. Our objective is now to find an appropriate continuum analog $\mathcal{I}$ of $\mathcal{P}$ such that $\mathcal{N}(\mathcal{I})$ is homologous to $\mathcal{N}(\mathcal{P})$. If $\mathcal{I}$ further satisfies the conditions of Corollary 2 this would mean that $\mathcal{N}(\mathcal{P})$ is homologous to $M$.

For any inextendible antichain $A$, there exists an infinity of Cauchy surfaces containing it. This makes the discrete-continuum correlation highly nonunique. Nevertheless, one can make a choice of $A$ such that this nonuniqueness becomes unimportant to recovering the continuum homology.

Let $\Sigma_{1}, \Sigma_{2}$ be a pair of Cauchy surfaces such that $A \subset \Sigma_{i}, i=1,2$. A spacing between the $\Sigma_{i}$ can be defined via a space-time interval of the type $\mathrm{I}\left(p_{1}, p_{2}\right)$, or $\mathrm{I}\left(q_{2}, q_{1}\right)$, for any $p_{i}, q_{i} \in \Sigma_{i}, i=1,2$ such that $p_{1}<<p_{2}$, and $q_{2}<<q_{1}$.

Claim 3: For any pair of Cauchy hypersurfaces $\Sigma_{1}, \Sigma_{2} \supset A$, the spacings between them lie in $S(A)$.

Proof: Without loss of generality, let there exist $p_{i} \in \Sigma_{i}, i=1,2$ such that $p_{1}<<p_{2}$. Then, by transitivity, for any $q \in \mathrm{I}\left(p_{1}, p_{2}\right), q \notin<\mathrm{I}^{ \pm}(A)$, since the $\Sigma_{i}$ are achronal. Further, if $q \in \partial \mathrm{J}^{ \pm}(A)$, by global hyperbolicity, this means that there exists an $a \in A$ such that $a \rightarrow q$ or $q \rightarrow a$. Assuming the former without loss of generality, since $q<<p_{2}$, this means that $a<<p_{2}$, which is a contradiction. Thus, $\mathrm{I}\left(p_{1}, p_{2}\right) \subset S(A)$.

Since $A$ is an inextendible antichain, $S(A)$ must be made up entirely of voids, i.e., $\Phi(C) \cap S(A)=\emptyset$. Thus, for any pair of $\Sigma_{i}$ 's, the spacings between them must be empty of causal set elements. Voids of volume $V$ occur with probability $P_{0}(V)=\exp ^{-V / V_{c}}$. Thus, the probability for a region of volume $V \gg V_{c}$ to be a spacing (and hence a void) is $\ll 1$. Given some $\Sigma_{i} \supset A$, the probability for any interval $I\left(p_{i}, q\right)$ to its future or $I\left(r, p_{i}\right)$ to its past of volume $V$ to be a spacing is then roughly limited by the number $N$ of such independent intervals. The probability that at least one of these intervals be a spacing is then $N P_{0}(V)$, which for $N$ sufficiently small, or $\Sigma$ sufficiently compact, is still $\ll 1$ for $V \gg V_{c}$.

Even for a space-time with a "sufficiently" compact $\Sigma$, it may be possible, although not probable, to pick from $\Phi(C)$ an inextendible antichain $A$ such that the volume of the spacings between the $\Sigma_{i} \supset A$ is not of $\mathcal{O}\left(V_{c}\right)$. We will henceforth only restrict to those $A$, for which this is not the case, i.e., the spacings are all of order $V_{c}$. This ensures that the Cauchy hypersurfaces containing such an $A$ differ from each other by a relative volume of $\mathcal{O}\left(V_{c}\right)$ and hence may be considered "equivalent" to each other at scales $\gg V_{c}$. We may therefore pick a $\Sigma$ from this set with the largest convexity radius $\widetilde{\nabla}$ and directly assign $\widetilde{v}$ to $A$.

We now explore the obvious analogy between the thickened antichain $\mathrm{T}_{\mathrm{n}}(A)$ for a likely $A$ and the thickened region, $M_{\mathrm{v}}$ sandwiched between $\Sigma \supset A$ and $\Sigma_{\mathrm{v}}$, where $\mathrm{v}=\mathrm{n} V_{c}$. Corollary 1 tells us that for $0<\mathrm{v}<\widetilde{\mathrm{v}}, \mathcal{N}\left(\mathcal{I}_{\mathrm{v}}\right)$ is homologous to the space-time. While the upper bound $\widetilde{v}$ obtains from continuum considerations, in the discrete context, one also expects a lower bound $\mathrm{v}_{0}$ arising from the discreteness scale. Indeed, such a lower bound makes its appearance in our numerical investigations. For example, for $\mathrm{v} \sim V_{c}$, there is a high probability for voids which "cut through" $M_{\mathrm{v}}$ in a swiss-cheese-like fashion. If $\mathrm{M}=\left\{m_{i}\right\}$ are the maximal elements of $\mathrm{T}_{\mathrm{n}}(A)$, the set of shadows $\left\{\sigma\left(m_{i}\right)\right\}$ on $\Sigma$ will most likely not cover $\Sigma$ for $\mathrm{n} \sim 1\left(\mathrm{v} \sim V_{c}\right)$. In other words, $M_{\mathrm{v}}$ is "too thin" to be a good approximation to $\mathrm{T}_{n}(A)$. Thus $\mathcal{I} \equiv\left\{\mathrm{I}\left(\Sigma, m_{i}\right)\right\}$ does not satisfy the conditions of Corollary 2 , so that $\mathcal{N}(\mathcal{I})$ need not be homologous to the space-time. Thus, it seems necessary to impose the condition that $\mathrm{v} \gg V_{c}$. Clearly, this is not sufficient for obtaining an $\mathcal{N}(\mathcal{I})$ homologous to the space-time since $\widetilde{\mathrm{v}}$ itself may be $\mathcal{O}\left(V_{c}\right)$ so that there exists no $\mathrm{v} \gg V_{c}$ with $\mathrm{v}<\widetilde{\mathrm{v}}$, and hence the conditions of Corollary 2 are not satisfied. Hence we must further restrict to inextendible antichains $A$ for which $\tilde{\mathrm{v}} \gg V_{c}$. 


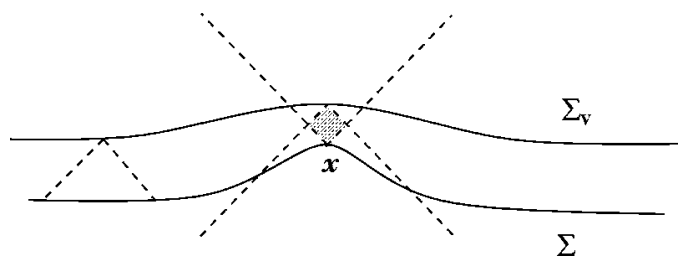

FIG. 3. The largest interval between a given $x \in \Sigma$ and a point in $\Sigma_{\mathrm{v}}$.

$M_{\mathrm{v}}$ can also fail to be a good continuum approximation of $\mathrm{T}_{\mathrm{n}}(A)$, even though $\widetilde{\mathrm{v}}>\mathrm{V} \gg V_{c}$, since it could be too thin in local patches. For example, if the extrinsic curvature at a point on $\Sigma$ is relatively large in comparison with other points in its neighborhood, the spacings between points on $\Sigma$ and $\Sigma_{\mathrm{v}}$ may still be of order $V_{c}$ even for $\mathrm{v} \gg V_{c}$. Figure 3 provides an illustration of this. Let us define

$$
\alpha_{x}(\mathrm{v}) \equiv \sup _{y \in \Sigma_{\mathrm{v}}} \operatorname{vol}(\mathrm{I}(x, y)), \quad x \in \Sigma,
$$

which is the size of the largest space-time interval between a given $x \in \Sigma$ and any $y \in \Sigma_{\mathrm{v}}$. If $\alpha(x) \sim V_{c}$, then $M_{\mathrm{V}}$ is once again too thin at $x$ and not a good continuum approximation to $\mathrm{T}_{\mathrm{n}}(A)$. Again, the probability of a void at $x$ which cuts through $M_{\mathrm{v}}$ is high. On the other hand, if $\alpha_{x}(\mathrm{v})$ $\gg V_{c}$ (which implies $\mathrm{V} \gg V_{c}$ ) for all $x \in \Sigma$, then such voids are less probable. We may therefore define the lower bound $\mathrm{v}_{0}$ on $\mathrm{v}$ such that $\forall \mathrm{v}>\mathrm{v}_{0}, \alpha_{x}(\mathrm{v}) \gg V_{c}, \forall x \in \Sigma$.

In order to obtain the high probability discrete-continuum correspondence for the nerves, we will find it necessary to impose a very strong separation of scales, $V_{c} \ll \mathrm{v}_{0} \ll \mathrm{v} \ll \widetilde{\mathrm{V}}$. This may be too stringent a requirement in practice, but it allows us to make our arguments in as simple and general a language as possible. It is useful to see if such a separation of scales lies within physically reasonable bounds. We can assume that the continuum description is valid at least down to length scales $l_{c}=10^{-25} \mathrm{~m}$, which gives us a cutoff volume of $V_{c}=10^{-100} \mathrm{~m}^{4}$. Let $\alpha_{x}\left(\mathrm{v}_{0}\right)$ $\sim 10^{12} V_{c}$ which has an associated length scale of $10^{-22} \mathrm{~m}$. A conservative choice for $\widetilde{v}$, which corresponds to the shortest scales at which spatial intrinsic and extrinsic curvature effects become important, is the nuclear scale $\sim 10^{40} V_{c}$. Thus, a separation of scales $10^{40} V_{c} \gg \mathrm{V} \gg 10^{12} V_{c} \gg V_{c}$ is possible within reasonable physical bounds, i.e., far above the Planck scale and far below the nuclear scale.

We have therefore placed fairly stringent requirements on the inextendible antichain $A$ with respect to its faithful embedding into the space-time $(M, g)$ at a fixed density $V_{c}^{-1}$. The obvious question is how to identify such $A$ in $C$ without reference to the embedding $\Phi(C)$ in $(M, g)$. However, this requires a better understanding of intrinsic geometrical information in the causal set than we presently have and is under current investigation. ${ }^{21}$ We instead adopt the following, mathematically equivalent, approach. Starting with a given $(M, g)$, we allow the sprinkling density to vary such that any $M_{\mathrm{v}}$ with $\mathrm{v}<\widetilde{\mathrm{v}}$ satisfies the condition $\mathrm{v}>\mathrm{v}_{0} \gg V_{c}$. Thus, we pick only those $A$ 's from $\Phi(C)$ satisfying this separation of scales.

Given an $A$ satisfying these above conditions, with associated $\widetilde{v}=\widetilde{n} V_{c}, \mathrm{v}_{0}=n_{0} V_{c}$, consider its thickening, $\mathrm{T}_{\mathrm{n}}(A)$, where $\tilde{\mathrm{n}} \gg \mathrm{n} \gg \mathrm{n}_{0} \gg 1$. For any $y \in \mathrm{J}^{+}(\Sigma)$, define $\mathrm{v}(y) \equiv \operatorname{vol}(\mathrm{I}(\Sigma, y))$ and $\mathrm{n}(y)$ $\equiv|\operatorname{IFut}(A) \cap \operatorname{IPast}(y)|$.

We now list probabilities for certain occurrences which will subsequently be used to quantify the "high probability" correspondence between the continuum and the discrete homologies. The probability for a void of volume $V^{\prime} \gg V_{c}$ is

$$
\mathrm{P}_{0} \equiv \exp ^{-V^{\prime} / V_{c}} \ll 1
$$

The probability for a region of volume $v_{0}$ to have $n$ sprinkled points with $n \gg n_{0} \gg 1$ is 


$$
\mathrm{P}_{1} \equiv \sum_{k=\mathrm{n}}^{\infty} \frac{1}{k !} \exp ^{-\mathrm{n}_{0}}\left(\mathrm{n}_{0}\right)^{k}=1-\frac{\Gamma\left(\mathrm{n}, \mathrm{n}_{0}\right)}{\Gamma(\mathrm{n})} \ll 1,
$$

where $\Gamma(x, y)$ is the incomplete gamma function. This is the probability of an "overstuffed" region. The probability for a region of volume $\widetilde{\nabla}=\tilde{n} V_{c}$ to contain $n$ sprinkled points with $1 \ll n \ll \tilde{n}$ is

$$
\mathrm{P}_{2} \equiv \sum_{k=0}^{\mathrm{n}} \frac{1}{k !} \exp ^{-\tilde{\mathrm{n}}_{0}}\left(\widetilde{\mathrm{n}}_{0}\right)^{k}=\frac{\Gamma(\mathrm{n}+1, \widetilde{\mathrm{n}})}{\Gamma(\mathrm{n}+1)} \ll 1
$$

This is the probability of an "understuffed" region. One will need to calculate the total probability for at least one such improbable situation (say, a void of volume $\gg V_{c}$ ) to occur anywhere in the thickened space-time region $M_{\mathrm{v}_{0}}$. Since the voids can overlap, one can set an upper bound on the probability by considering nonoverlapping regions, which can be constructed from the nonoverlapping shadows $\sigma_{i}$ from events on $\Sigma_{\mathrm{v}_{0}}$. For a given $A$ and associated $\Sigma$, the volume of a spacetime interval $\mathrm{I}(\Sigma, p)$ for any $p \in \Sigma_{\mathrm{v}_{0}}$ is $\mathrm{v}_{0}$. Since $\operatorname{vol}\left(M_{\mathrm{v}_{0}}\right)$ is roughly $\sim \mathrm{v}_{0}^{1 / n} \operatorname{vol}(\Sigma)$, where $\mathfrak{n}$ is the space-time dimension (since these intervals lie in CNN's where the deviation from flatness is small, this suffices to give a rough order of magnitude estimate, which is all we require), the number $\mathfrak{N}$ of such nonoverlapping regions in $M_{\mathrm{v}_{0}}$ is

$$
\mathfrak{N} \sim \operatorname{vol}(\Sigma) v_{0}^{-(\mathfrak{n}-1) / \mathfrak{n}} .
$$

For $\mathrm{v}>\mathrm{v}_{0}$, the corresponding number of independent regions is $\mathfrak{N}_{\mathrm{v}}<\mathfrak{N}$ and hence we may use $\mathfrak{N}$ as an upper bound for all such $v$. Instead of encumbering ourselves with notation, we will then simply use $\mathfrak{N}$ itself as an adequate measure of the number of nonoverlapping regions, even for $\mathrm{V} \gg \mathrm{v}_{0}$. If $\mathfrak{N}$ is very large, then $\Sigma$ is correspondingly large and the total probability for a rare event may be non-negligible. Thus, we quantify our requirement for $\Sigma$ to be sufficiently compact by

$$
\mathfrak{N P}_{0} \ll 1, \quad \mathfrak{N P} P_{1} \ll 1, \quad \mathfrak{N P} P_{2} \ll 1 .
$$

It is instructive at this point to check how restrictive this is for our present universe. If we take $A$ to lie in a homogeneous isotropic $\Sigma$, assuming $V_{c}=V_{p}$, roughly, $10^{180} \leqslant \mathfrak{N} \leqslant 10^{240}$. The probability that there are no voids of volume $\gg 10^{4} V_{p}$, say, is then $<10^{240} \times \exp ^{-10000} \sim \exp ^{-9447} \ll 1$.

We now demonstrate some results which will simplify the proof of our final Lemma.

Claim 4: For any $x \in \Sigma$, there exists at least one point in $\mathrm{I}^{+}(x)$ which belongs to $\mathrm{T}_{\mathrm{n}}(A)$ with high probability.

Proof: For any $x \in \Sigma, \alpha_{x}\left(\mathrm{v}_{0}\right) \gg V_{c}$, so that $\operatorname{vol}\left(\mathrm{I}\left(x, \Sigma_{\mathrm{V}_{0}}\right)\right) \gg V_{c}$. The probability of finding at least one sprinkled point in $\mathrm{I}\left(x, \Sigma_{\mathrm{v}_{0}}\right)$ is therefore $1-\mathrm{P}_{0}$, where $\mathrm{P}_{0} \ll 1$. The probability of finding at least one void in $M_{\mathrm{v}_{0}}$ of this kind is then $\sim \mathfrak{N P}_{0} \ll 1$.

For any $p \in \mathrm{I}\left(x, \Sigma_{\mathrm{v}_{0}}\right), \mathrm{v}(p)<\mathrm{v}_{0}$. The probability that it has $\mathrm{n}(p)>\mathrm{n} \gg \mathrm{n}_{0}$ is less than $\mathrm{P}_{1} \ll 1$. Hence, the probability for at least one of the $\mathfrak{N}$ nonoverlapping intervals to have such a point is less than $\mathfrak{N P} P_{1}$. The total (joint) probability for this occurrence is then $\left(1-\mathfrak{N P} P_{0}\right) \times \mathfrak{N P} P_{1} \sim \mathfrak{N P} P_{1}$ $\ll 1$. Conversely, the probability for every $x \in \Sigma$ to contain at least one point in its future belonging to $\mathrm{T}_{\mathrm{n}}(A)$ is $1-\mathfrak{N P}_{1} \sim 1$.

Note: The above claim also holds for any $y \in \mathrm{I}^{+}(\Sigma)$ such that $\mathrm{v}(y)=\mathcal{O}\left(V_{c}\right)$, since $\alpha_{y}\left(\mathrm{v}_{0}\right) \gg V_{c}$.

Claim 5: Let $\mathrm{M}$ be the set of maximal elements in $\mathrm{T}_{\mathrm{n}}(A)$. Then with high probability, none of the elements $e_{i} \in \Phi(C)$ with $\mathrm{v}\left(e_{i}\right)=\mathcal{O}\left(V_{c}\right)$ belong to $\mathrm{M}$.

Proof: Let $e_{1} \in \operatorname{Fut}(A)$ such that $\mathrm{v}\left(e_{1}\right)=\mathcal{O}\left(V_{c}\right)$. Then, $\mathrm{v}\left(e_{1}\right) \ll \mathrm{v}_{0}$ and hence $\mathrm{I}\left(e_{1}, \Sigma_{\mathrm{v}_{0}}\right) \gg V_{c}$. Then $\mathrm{I}\left(e_{1}, \Sigma_{\mathrm{v}_{0}}\right) \cap \mathrm{T}_{\mathrm{n}}(A)=\emptyset$ occurs with probability $\sim \mathrm{P}_{1} \ll 1$ from Claim 4 . Therefore $e_{1}$ is not a maximal element with probability $>1-\mathrm{P}_{1}$. The number of $e_{i}$ with $\mathrm{v}\left(e_{i}\right)=\mathcal{O}\left(V_{c}\right) \Rightarrow \mathrm{I}\left(e_{i}, \Sigma_{\mathrm{v}_{0}}\right) \gg V_{c}$ which are non-overlapping is $\sim \mathfrak{N}$, and hence the joint probability that none of them is a maximal element is $\sim 1-\mathfrak{N P}_{1} \sim 1$.

Claim 6: Every $x \in \Sigma$ lies in a shadow $\sigma_{i} \equiv \mathrm{I}^{-}\left(m_{i}\right) \cap \Sigma$ with high probability.

Proof: The proof of this is immediate from Claim 4. Namely, the probability that there exists at least one $x \in \Sigma$ which does not lie in a shadow is $\mathfrak{N P}_{1}$ and hence with probability $1-\mathfrak{N P} P_{1}$ 
$\sim 1$ every $x$ lies in a shadow.

Claim 7: Let $\mathcal{I}=\left\{\mathrm{I}_{i}\right\}$, with $\mathrm{I}_{i} \equiv \mathrm{I}\left(\Sigma, m_{i}\right) . \mathcal{N}(\mathcal{I})$ is homotopic to $M$ with high probability.

Proof: From Claim 6 with probability $1-\mathfrak{N P}{ }_{1}, \mathcal{S} \equiv\left\{\sigma_{i}\right\}$ is a cover of $\Sigma$. Moreover, with probability $1-\mathfrak{N P}_{2}, \mathrm{v}\left(m_{i}\right)<\widetilde{\nabla}$, so that the joint probability for $\mathcal{S}$ to be a convex cover of $\Sigma$ is $\sim 1-\mathfrak{N}\left(\mathrm{P}_{1}+\mathrm{P}_{2}\right)$. Thus, from Corollary $2, \mathcal{N}(\mathcal{S})$ and $\mathcal{N}(\mathcal{I})$ are both homotopic to $M$ with probability $\sim 1-\mathfrak{N}\left(\mathrm{P}_{1}+\mathrm{P}_{2}\right) \sim 1$.

We are now in a position to begin the discrete-continuum comparison.

Claim 8: Define the discrete collection of sets $\mathcal{P}=\left\{P_{i}\right\}, P_{i} \equiv \operatorname{Fut}(A) \cap \operatorname{IPast}\left(m_{i}\right)$. There is a one-to-one and onto map from $\mathcal{I}$ to $\mathcal{P}$. Hence the vertices of $\mathcal{N}(\mathcal{P})$ and $\mathcal{N}(\mathcal{I})$ are in one-to-one correspondence.

Note: The $P_{i}$ does not include the elements of $A_{i}$ in this definition and hence differ from the $P_{i}$ of Sec. III. The reason to adopt this modified choice comes from the fact that unlike in the continuum, there is no natural distinction between elements which are causally related and those that are strictly chronologically related in a causal set. In particular, Claim 9 would not be valid without this modification.

Proof: For every $m_{i}$ there exists a unique $P_{i}$, since the set of maximal points $\mathrm{M}$ forms an antichain (which need not be inextendible) and hence $m_{i} \in P_{j}$ iff $m_{i}=m_{j}$. Moreover, because the space-time is distinguishing, every $m_{i}$ has a unique $\mathrm{I}^{-}\left(m_{i}\right)$ and hence a unique $\mathrm{I}_{i}$.

This bijection between the vertices of $\mathcal{N}(\mathcal{P})$ and $\mathcal{N}(\mathcal{I})$ allows us to label the vertices as $m_{0}, m_{1}, \ldots, m_{|\mathbf{M}|}$. Therefore the largest possible dimension of a simplex in $\mathcal{N}(\mathcal{I})$ or $\mathcal{N}(\mathcal{P})$ is $|\mathbf{M}|$.

Claim 9: $\mathcal{N}(\mathcal{P})$ is a subcomplex of $\mathcal{N}(\mathcal{I})$.

Proof: For any $p \in P_{i_{1} \ldots i_{k}} \equiv P_{i_{1}} \cap \cdots \cap P_{i_{k}} \neq \emptyset, p<m_{i_{j}}$ for all $j=1, \ldots, k$. Since $p \in \mathrm{I}^{+}(\Sigma), \exists x$ $\in \mathrm{I}^{+}(\Sigma)$ such that $p \in \mathrm{I}^{+}(x)$, so that $m_{i_{j}} \in \mathrm{I}^{+}(x)$, for all $j=1, \ldots, k$, and hence $p \in \mathrm{I}_{i_{1} \ldots i_{k}}$ $\equiv \mathrm{I}_{i_{1}} \cap \ldots \cap \mathrm{I}_{i_{k}} \neq \emptyset$. Note that without the modified definition of the $P_{i}$ this would no longer be true. For example, if the intersection is nonempty only on the light cones and on $A$, then any $p$ $\in A$ and does not lie in an intersection of $\mathrm{I}_{i}$ 's.

However, $\mathcal{N}(\mathcal{I})$ is not in general a subcomplex of $\mathcal{N}(\mathcal{P})$ since there may exist nonempty intersections $\mathrm{I}_{i_{1} \ldots i_{k}} \neq \emptyset$ with $\operatorname{vol}\left(\mathrm{I}_{i_{1} \ldots i_{k}}\right)=\mathcal{O}\left(V_{c}\right)$. There is then a high probability for such intersections to be voids, i.e., $P_{i_{1} \ldots i_{k}}=\emptyset$. If this is the case, then there is no $k$-simplex in $\mathcal{N}(\mathcal{P})$ which maps to this particular $k$-simplex in $\mathcal{N}(\mathcal{I})$. In other words, $\mathcal{N}(\mathcal{P})$ and $\mathcal{N}(\mathcal{I})$ need not be homotopic to each other.

Despite this, we now show that $\mathcal{N}(\mathcal{P})$ and $\mathcal{N}(\mathcal{I})$ are homologous to each other with high probability, since $\mathcal{N}(\mathcal{P})$ is an adequate subcomplex of $\mathcal{N}(\mathcal{I})$ with high probability. We begin by establishing some notation and reminding the reader of some basics of algebraic topology. ${ }^{18}$

We begin by setting an orientation on the simplicial complex $\mathcal{N}(\mathcal{I})$, so that the set of vertices $\left\{m_{i}\right\}=\mathrm{M}$ is ordered as $m_{0} \rightarrow m_{1} \rightarrow \cdots \rightarrow m_{|\mathrm{M}|}$. Let $C_{q}(\mathcal{N}(\mathcal{I}))$ be the free Abelian group with basis $\mathfrak{B}_{q} \equiv\left\{\hat{b}_{q}^{(\alpha)}\right\}$, where the $\hat{b}_{q}^{(\alpha)}=\left(m_{s_{0}}, \ldots, m_{s_{q}}\right)$ are $q$-simplices in $\mathcal{N}(\mathcal{I})$, such that $\left(m_{s_{\pi(0)}}, \ldots, m_{s_{\pi(q)}}\right)$ $=\operatorname{sign}(\pi)\left(m_{s_{0}}, \ldots, m_{s_{q}}\right)$ for $\pi$ a permutation of the set $\{0,1, \ldots, q\}$. Elements of $C_{q}(\mathcal{N}(\mathcal{I}))$ are referred to as $q$-chains. For $q>|\mathrm{M}|, C_{q}(\mathcal{N}(\mathcal{I}))=\emptyset . Z_{q}(\mathcal{N}(\mathcal{I})) \subset C_{q}(\mathcal{N}(\mathcal{I}))$ are the simplicial $q$-cycles and $B_{q}(\mathcal{N}(\mathcal{I})) \subset C_{q}(\mathcal{N}(\mathcal{I}))$ the simplicial $q$-boundaries, so that the $q$ th homology group $H_{q}(\mathcal{N}(\mathcal{I})) \equiv Z_{q}(\mathcal{N}(\mathcal{I})) / B_{q}(\mathcal{N}(\mathcal{I}))$. It will be convenient to use the shortened notation $K \equiv \mathcal{N}(\mathcal{I})$, $C_{q} \equiv C_{q}(\mathcal{N}(\mathcal{I})), Z_{q} \equiv Z_{q}(\mathcal{N}(\mathcal{I})), B_{q} \equiv B_{q}(\mathcal{N}(\mathcal{I}))$ and denote with primes the associated sets for the simplicial complex $K^{\prime} \equiv \mathcal{N}(\mathcal{P})$.

Now, a $q$-simplex $\left(m_{s_{0}}, \ldots, m_{s_{q}}\right)$ is an element of $\mathfrak{B}_{q}$ iff the intersections $\mathrm{I}_{s_{0} \ldots s_{q}} \neq \emptyset$, and similarly, $\left(m_{s_{0}^{\prime}}, \ldots, m_{s_{q}^{\prime}}\right)$ is an element of $\mathfrak{B}_{q}^{\prime}$ iff $P_{\alpha_{0}^{\prime} \ldots \alpha_{q}^{\prime}} \neq \emptyset$. We will use the notation $\left(s_{0}, \ldots, s_{q}\right) \equiv\left(m_{s_{0}}, \ldots, m_{s_{q}}\right)$ and $\left.\left[s_{0}, \ldots, s_{q}\right] \equiv\right|_{s_{0} \ldots s_{q}}$, which also helps us switch more easily from sets

to simplices and back. As in standard notation a $q-1$-simplex $\left(s_{0}, \ldots, \hat{s}_{i}, \ldots, s_{q}\right)$ can be obtained from a $q$-simplex $\left(s_{0}, \ldots, s_{q}\right)$ by simply dropping the $i$ th vertex.

A subcomplex $K^{\prime}$ of a simplicial complex $K$ is said to be adequate if for all $q \geqslant 0$,

(1) if $z \in Z_{q}$, then there exists a $z^{\prime} \in Z_{q}^{\prime}$ with $z-z^{\prime} \in B_{q}$ and

(2) if $z^{\prime} \in Z_{q}^{\prime}$ and $z^{\prime}=\partial c$ for some $c \in C_{q+1}$, then there exists a $c^{\prime} \in C_{q+1}^{\prime}$ with $z^{\prime}=\partial c^{\prime}$. 
Lemma 3 (Rotman): If $K^{\prime}$ is an adequate subcomplex of $K$, then for every $q$, the map $z^{\prime}$ $+B_{q}^{\prime} \mapsto z^{\prime}+B_{q}$ is an isomorphism

$$
H_{q}\left(K^{\prime}\right) \simeq H_{q}(K) .
$$

Thus, our task is reduced to showing that $\mathcal{N}(\mathcal{P})$ is an adequate subcomplex of $\mathcal{N}(\mathcal{I})$.

We will begin by defining a growth of a $q+1$-chain $\tau$ from a given $q$-simplex, $\mathcal{F}_{0}$ $\equiv\left(s_{0}, \ldots, s_{q}\right)$. We describe this construction in some detail, since it is crucial to the proof of the main results of this section. Starting with some $\mathcal{F}_{0}$, let there exist an $s_{q+1}$ such that there exists a nonempty $q+1$-simplex

$$
\tau_{1} \equiv\left(s_{0}, \ldots, s_{q}, s_{q+1}\right) \neq \emptyset .
$$

We have thus "grown" a $q+1$-chain (in this case simply a $q+1$-simplex) from $\mathcal{F}_{0}$. The boundary of $\tau_{1}$ then consists of the set of $q$-simplices,

$$
\mathcal{F}_{1} \equiv\left\{\left(s_{0}, \ldots, \hat{s}_{i}, \ldots, s_{q+1}\right)\right\}, \quad \forall i \in[0, q],
$$

and the original $q$-simplex $\mathcal{F}_{0}$. We may stop the growth here.

However, if for some $i_{1} \in[0, q]$, there exist an $s_{q+2}^{\left(i_{1}\right)} \neq s_{i_{1}}$ such that

$$
\tau_{2}^{\left(i_{1}\right)} \equiv\left(s_{0}, \ldots, \hat{s}_{i_{1}}, \ldots, s_{q+1}, s_{q+2}^{\left(i_{1}\right)}\right) \neq \emptyset,
$$

then the growth can be continued to obtain a larger $q+1$-chain containing $\tau_{1}$. The $q+1$-simplex $\tau_{2}^{\left(i_{1}\right)}$ has the set of boundary $q$-simplices

$$
\mathcal{F}_{2}^{\left(i_{1}\right)} \equiv\left\{\left(s_{0}, \ldots, \hat{s}_{i_{1}}, \ldots, \hat{s}_{j}, \ldots, s_{q+1}, s_{q+2}^{\left(i_{1}\right)}\right)\right\}, \quad \forall j \in\left[0, \ldots \hat{i}_{1}, \ldots q+1\right],
$$

along with $\left(s_{0}, \ldots, \hat{s}_{i_{1}}, \ldots, s_{q+1}, \hat{s}_{q+2}^{\left(i_{1}\right)}\right) . \tau_{1}$ and $\tau_{2}^{\left(i_{1}\right)}$ then share the single $q$-simplex along their boundary

$$
\Lambda^{\left(i_{1}\right)} \equiv\left(s_{0}, \ldots, \hat{s}_{i_{1}}, \ldots, s_{q+1}, \hat{s}_{q+2}^{\left(i_{1}\right)}\right),
$$

and the boundary of the $q+1$-chain $\tau_{1}+\tau_{2}^{\left(i_{1}\right)}$ consists of the set of $q$-simplices

$$
\mathcal{F}_{0} \cup \mathcal{F}_{1} \cup \mathcal{F}_{2}^{\left(i_{1}\right)}-\Lambda^{\left(i_{1}\right)} .
$$

Again, we could stop the growth here along the $i_{1}$ th branch.

If for a given $i_{1}$, there is an $i_{2} \in\left[0, \ldots, \hat{i}_{1}, \ldots, q+1\right]$ such that there exists an $s_{q+3}^{\left(i_{1} i_{2}\right)} \neq s_{i_{1}}, s_{i_{2}}$ such that

$$
\tau_{3}^{\left(i_{1} i_{2}\right)} \equiv\left(s_{0}, \ldots, \hat{s}_{i_{1}}, \ldots, \hat{s}_{i_{2}}, \ldots, s_{q+1}, s_{q+2}^{\left(i_{1}\right)}, s_{q+3}^{\left(i_{1} i_{2}\right)}\right) \neq \emptyset,
$$

then we may continue the growth in the $i_{1} i_{2}$ branch. The $q+1$-simplex $\tau_{3}^{\left(i_{1} i_{2}\right)}$ consists of the set of $q$-simplices on its boundary

$$
\mathcal{F}_{3}^{\left(i_{1} i_{2}\right)} \equiv\left\{\left(s_{0}, \ldots, \hat{s}_{i_{1}}, \ldots, \hat{s}_{i_{2}}, \ldots, \hat{s}_{k}, \ldots, s_{q+3}^{\left(i_{1} i_{2}\right)}\right)\right\}, \quad \forall j \in\left[0, \ldots, \hat{i}_{1}, \ldots, \hat{i}_{2}, \ldots, q+2\right],
$$

along with $\left(s_{0}, \ldots, \hat{s}_{i_{1}}, \ldots, \hat{s}_{i_{2}}, \ldots, \hat{s}_{q+3}^{\left(i_{1} i_{2}\right)}\right) . \tau_{2}^{\left(i_{1}\right)}$ and $\tau_{3}^{\left(i_{1} i_{2}\right)}$ then share a single $q$-simplex along their boundary

$$
\Lambda^{\left(i_{1} i_{2}\right)} \equiv\left(s_{0}, \ldots, \hat{s}_{i_{1}}, \ldots, \hat{s}_{i_{2}}, \ldots, \hat{s}_{q+3}^{\left(i_{1} i_{2}\right)}\right) .
$$

The boundary of the $q+1$-chain $\tau_{1}+\tau_{2}^{\left(i_{1}\right)}+\tau_{3}^{\left(i_{1} i_{2}\right)}$ then contains the set of $q$-simplices

$$
\mathcal{F}_{0} \cup \mathcal{F}_{1} \cup \mathcal{F}_{2}^{\left(i_{1}\right)} \cup \mathcal{F}_{3}^{\left(i_{1} i_{2}\right)}-\Lambda
$$




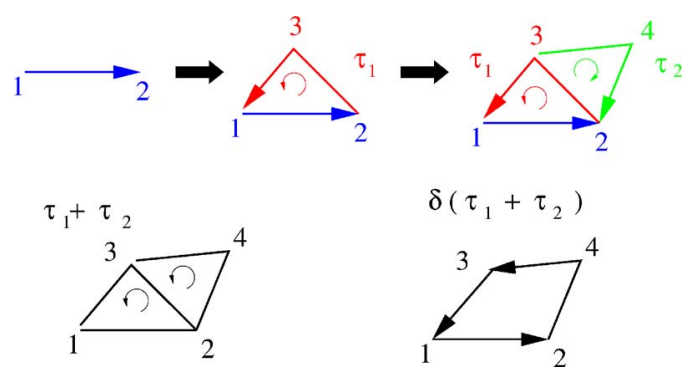

FIG. 4. (Color online) The growth of a 2-chain from a 1-simplex.

In this manner, we may carry on the growth of the $q+1$ chain along each branch up to the point desired or until it cannot be grown further. For a finite simplicial complex this will end in a finite number of steps. The final $q+1$-chain is

$$
\tau \equiv \tau_{1}+\left[\sum_{i_{1} \in G_{1}}\left[\tau_{2}^{\left(i_{1}\right)}+\sum_{i_{2} \in G_{12}}\left[\tau_{3}^{\left(i_{1} i_{2}\right)} \ldots\right]\right]\right],
$$

whose boundary contains the set of $q$-simplices

$$
\mathcal{F} \equiv \mathcal{F}_{0} \cup\left[\mathcal{F}_{1}\left[\cup_{i_{1} \in G_{1}} \mathcal{F}_{2}^{\left(i_{1}\right)}\left[\underset{i_{2} \in G_{12}}{\cup} \mathcal{F}_{3}^{\left(i_{1} i_{2}\right)} \ldots\right]\right]\right]-\Lambda,
$$

where

$$
\Lambda \equiv\left[\cup_{i_{1} \in G_{1}} \Lambda^{\left(i_{1}\right)}\left[\underset{i_{2} \in G_{12}}{\cup} \Lambda^{\left(i_{1} i_{2}\right)} \ldots\right]\right],
$$

and $G_{1}$ is the set of $i_{1}, G_{12}$ the set of $i_{2}$ 's in the $i_{1}$ branch, etc. Important in this growth is the set $\Lambda$ which has been "removed" from the boundary of $\tau$. We illustrate this for the simple case of the growth of a 2-chain from a 1-simplex in Fig. 4. We will now use this growth process to eliminate the set $\Lambda$ of $q$-simplices which have volume $\mathcal{O}\left(V_{c}\right)$ or are "thin." We will say that $\left[s_{0}, \ldots, s_{q}\right]$ $\neq \emptyset$ is thin if $\forall x \in\left[s_{0}, \ldots, s_{q}\right], \mathrm{v}(x)=\mathcal{O}\left(V_{c}\right)$ and "fat" if there exists a maximal element $x$ $\in \overline{\left[s_{0}, \ldots, s_{q}\right]}$ such that $\mathrm{v}(x) \gg V_{c}$.

Claim 10: For every $\mathcal{F}_{0} \equiv\left(s_{0}, \ldots, s_{q}\right)$ such that $\operatorname{vol}\left[s_{0}, \ldots, s_{q}\right]=\mathcal{O}\left(V_{c}\right) \neq \emptyset$, there exists, with high probability, a q+1-chain $\tau$ grown from $\mathcal{F}_{0}$ as in Eq. (24) with boundary made up of the set of q-simplices $\mathcal{F}$ as in Eq. (25), all of which correspond to fat sets, save $\mathcal{F}_{0}$ itself.

Proof: Since $\left[s_{0}, \ldots, s_{q}\right]$ is thin, with probability $P(q+1) \sim 1-\mathrm{P}_{1}$ there exists an $m_{s_{q+1}}$ which lies in the future of a maximal event in $\overline{\left[s_{0}, \ldots, s_{q}\right]}$. This follows from Claim 4. By set inclusion, $\left[s_{0}, \ldots, s_{q}, s_{q+1}\right] \subseteq\left[s_{0}, \ldots, s_{q}\right]$ and moreover, $\left[s_{0}, \ldots, s_{q}, s_{q+1}\right] \neq \emptyset$ by transitivity, so that

$$
\tau_{1}=\left(s_{0}, \ldots, s_{q}, s_{q+1}\right) \neq \emptyset .
$$

This is the first stage of the growth process. Now, if there exists no $i_{1}$ such that $\left[s_{0}, \ldots, \hat{s}_{i_{1}}, \ldots, s_{q+1}\right]$ is thin, then we may stop the growth process. The boundary of $\tau \equiv \tau_{1}$ is made up of the set of $q$-simplices $\mathcal{F}_{1} \cup \mathcal{F}_{0}(15)$ which are all fat, save $\mathcal{F}_{0}$.

Assume otherwise, i.e., let there be an $i_{1} \neq q+1$ such that $\left[s_{0}, \ldots, \hat{s}_{i_{1}}, \ldots, s_{q+1}\right]$ is thin. With probability $P(q+2) \sim 1-\mathrm{P}_{1}$, there exists an $m_{s_{q+2}}^{\left(i_{1}\right)}$ which lies to the future of a maximal event of $\left[s_{0}, \ldots, \hat{s}_{i_{1}}, \ldots, s_{q+1}\right]$, such that

$$
\left[s_{0}, \ldots, \hat{s}_{i_{1}}, \ldots, s_{q+1}\right] \supseteq\left[s_{0}, \ldots, \hat{s}_{i_{1}}, \ldots, s_{q+2}^{\left(i_{1}\right)}\right] \neq \emptyset,
$$

so that 


$$
\tau_{2}^{\left(i_{1}\right)}=\left(s_{0}, \ldots, \hat{s}_{i_{1}}, \ldots, s_{q+2}^{\left(i_{1}\right)}\right) \neq \emptyset .
$$

Now, choose the maximal event such that $m_{s_{q+2}}^{\left(i_{1}\right)} \neq m_{s_{i_{1}}}$. If such a choice of $m_{s_{q+2}}^{\left(i_{1}\right)}$ is not possible, then we have reached the end of the growth. Thus, we prevent ourselves from picking up a vertex that we previously dropped along any given branch of the growth. Then $\tau \equiv \tau_{1}+\tau_{2}^{\left(i_{1}\right)}$ has a boundary made up of the simplices (19).

The total probability for $m_{s_{q+2}}^{\left(i_{1}\right)}$ and $m_{s_{q+1}}$ to both occur is then bounded from below by $P(q$ +1) $P(q+2) \sim 1-2 \mathrm{P}_{1}$.

Again, if there exists no $i_{2}$ such that $\left[s_{0}, \ldots, \hat{s}_{i_{1}}, \ldots, \hat{s}_{i_{2}}, \ldots, s_{q+2}^{\left(i_{1}\right)}\right]$ is thin, then we may again stop the growth process and $\tau$ is the $q+1$-chain $\tau_{1}+\tau_{2}^{\left(i_{1}\right)}$ whose boundary is made up of the set of $q$-simplices (19), all of which are fat, save $\mathcal{F}_{0}$.

Since there are only a finite number of elements of $M$, this process must stop in a finite number of steps. If the end of the growth along any of the branches yields a final $m_{s_{q+k}}^{\left(i_{1} i_{2} \ldots i_{r}\right)}$ such that there exist sets $\left[s_{0}, \ldots, \hat{s}_{i_{1}}, \ldots, \hat{s}_{i_{r}}, s_{q+r}^{\left(i_{1} \ldots i_{r}\right)}\right]$ which are not fat, then this occurs with probability $\mathrm{P}_{1} \ll 1$. Thus, with probability greater than $1-r \mathrm{P}_{1} \sim 1$, a given "final" intersection along a branch $i_{1} i_{2}, \ldots, i_{r}$ is fat. Since there is a maximum of $\mathfrak{N}$ nonoverlapping regions, this is true along all of the branches with probability $>1-\mathfrak{N P} P_{1} \sim 1$.

The end result of this growth thus yields a $q+1$ chain $\tau(24)$ whose boundary is a collection of $q$-simplices (25), which are all fat, save $\mathcal{F}_{0}$, with high probability.

Lemma 4: There exists an isomorphism $H_{q}(\mathcal{N}(\mathcal{P})) \simeq H_{q}(M)$ with high probability.

Proof: Let $K^{\prime} \equiv \mathcal{N}(\mathcal{P})$ and $K \equiv \mathcal{N}(\mathcal{I})$. $C_{0}$ is isomorphic to $C_{0}^{\prime}$ since the vertices of the complexes $K$ and $K^{\prime}$ are the same, by Claim 8 . Thus, $\mathfrak{B}_{0} \equiv \mathfrak{B}_{0}^{\prime}$ and any $z \in Z_{0}$ lies in $Z_{0}^{\prime}$ and vice versa, and every $z \in B_{0}$ lies in $B_{0}^{\prime}$, thus satisfying the two requirements for an adequate subcomplex, when $q=0$. Henceforth we assume that $q>0$. We show one by one that both requirements are satisfied by $\mathcal{N}(\mathcal{P})$ with high probability.

(i) If $z_{1} \in Z_{q}$ and $z_{1} \in B_{q}$, then for $z^{\prime}=\emptyset, z_{1}-z^{\prime} \in B_{q}$ and we are done. Also, if $z_{1} \in Z_{q}^{\prime}$, then setting $z^{\prime}=z_{1}$ gives us $z_{1}-z^{\prime}=\emptyset \in B_{q}$ and again, we are done.

Therefore, we only need to consider $z_{1} \in Z_{q}$ such that $z_{1} \notin B_{q}, z_{1} \notin Z_{q}^{\prime} . \mathfrak{B}_{q}^{\prime} \subset \mathfrak{B}_{q}$ is then a strict inclusion. Let $z_{1}=\sum_{\alpha=1}^{r} \mu_{\alpha} \hat{b}_{q}^{(\alpha)}$. Since $z_{1} \notin Z_{q}^{\prime}$, there exists a $\mu_{\alpha^{\prime}} \neq 0$, such that $\hat{b}_{q}^{\left(\alpha^{\prime}\right)} \notin \mathfrak{B}_{q}^{\prime}$. Now, $\hat{b}_{q}^{\left(\alpha^{\prime}\right)} \equiv\left(m_{s_{0}}, \ldots, m_{s_{q}}\right)$ is such that $\left[m_{s_{0}}, \ldots, m_{s_{q}}\right] \neq \emptyset$ is a void. If $\left[m_{s_{0}}, \ldots, m_{s_{q}}\right]$ is fat, then one has a void of volume $V \gg V_{c}$ which occurs with probability $\mathrm{P}_{0} \ll 1$ and is hence unlikely. The probability that there are no voids of volume $V \gg V_{c}$ to the future of $\Sigma$ is then $\sim 1-\mathfrak{N P} P_{0} \sim 1$. Hence, with probability $>1-\mathfrak{N P} P_{0}$ any such $\left[m_{s_{0}}, \ldots, m_{s_{q}}\right]$ is thin. From Claim 10, then with probability greater than $1-\mathfrak{N P}_{1}$, there exists a $q+1$-chain $\tau$ whose boundary $\partial \tau$ is made up of fat $q$-simplices, save for $\hat{b}_{q}^{\left(\alpha^{\prime}\right)}$. Moreover, any fat $q$-simplex in $\mathfrak{B}_{q}$ is, with probability $1-\mathrm{P}_{0}$, a basis element of $\mathfrak{B}_{q}^{\prime}$. Since these sets are not independent, the probability for all the fat $q$-simplices to be basis elements of $\mathfrak{B}_{q}^{\prime}$ is bounded from below by $1-r^{\prime} \mathrm{P}_{0}$, where $r^{\prime}$ is the number of all $\mu_{\alpha} \neq 0$, save one, and is such that $r^{\prime} \leqslant \mathfrak{N}$. Thus, for any basis element $\hat{b}_{q}^{\left(\alpha^{\prime}\right)} \notin \mathfrak{B}_{q}^{\prime}$, with probability $1-\mathfrak{N}\left(\mathrm{P}_{0}+\mathrm{P}_{1}\right)-r^{\prime} \mathrm{P}_{0}$ $\sim 1$ we can associate a $q+1$-chain $\tau$ whose boundary $\partial \tau$ is made up of basis elements that belong to $\mathfrak{B}_{q}^{\prime}$, save for $\hat{b}_{q}^{\left(\alpha^{\prime}\right)}$.

Then $z_{2}=z_{1}-(-1)^{q+1} \mu_{\alpha^{\prime}} \partial \tau \in Z_{q}$ and $z_{1}-z_{2} \in B_{q}$. Moreover, writing $z_{2}=\sum_{\alpha=1}^{r} \nu_{\alpha} \hat{b}_{q}^{(\alpha)}$ gives $\nu_{\alpha^{\prime}}$ $=0$. If this was the only basis element in $z_{1}$ not in $\mathfrak{B}_{q}^{\prime}$, then we are done. We may thus "weed out" all the thin intersections in $z_{1}$ iteratively, until we finally get a $z_{k}=\sum_{\alpha=1}^{r} \mu_{\alpha}^{\prime} \hat{b}_{q}^{(\alpha)}$ such that all for all $\mu_{\alpha} \neq 0$, the $\hat{b}_{q}^{(\alpha)}$ are fat.

Then, with probability of at least $1-\mathfrak{N}\left(\mathrm{P}_{1}+2 \mathrm{P}_{0}\right) \sim 1$ for every $z_{1} \in Z_{q}$ there is a $z^{\prime} \in Z_{q}^{\prime}$ such that $z_{1}-z^{\prime} \in B_{q}$.

(ii) Let $z \in Z_{q}^{\prime}$ and $z \in B_{q}$, i.e., $z=\partial c_{1}$ for some $c_{1} \in C_{q+1}$. If $c_{1} \in C_{q+1}^{\prime}$, then we are done. Let us assume otherwise, i.e., $c_{1} \notin C_{q+1}^{\prime}$. Then $\mathfrak{B}_{q+1}^{\prime} \subset \mathfrak{B}_{q+1}$ is a strict inclusion. 
Let $c_{1}=\sum_{\alpha=1}^{r} \mu_{\alpha} \hat{b}_{q+1}^{(\alpha)} \in C_{q+1}$. Then there exists a $\mu_{\alpha^{\prime}} \neq 0$ such that $\hat{b}_{q+1}^{\left(\alpha^{\prime}\right)} \notin \mathfrak{B}_{q}^{\prime}$. For a given $\hat{b}_{q+1}^{\left(\alpha^{\prime}\right)} \equiv\left(m_{s_{0}}, \ldots, m_{s_{q+1}}\right)$, according to Claim 10 , with probability $1-\mathfrak{N P} \mathrm{P}_{1}$, there exists a $q+2$-chain $\tau$ whose boundary $\partial \tau$ is made up of fat $q+1$-simplices, save for $\hat{b}_{q+1}^{\left(\alpha^{\prime}\right)}$.

Let $c_{2}=c_{1}-(-1)^{q+2} \mu_{\alpha^{\prime}} \partial \tau \in C_{q+1}$. Clearly, $\partial c_{1}=\partial c_{2}$. Hence, as in (i) the thin simplices in $c_{1}$ may be weeded out until one obtains a $c^{\prime}$ made up of only fat basis elements.

The analysis is similar to that in (i) and hence with probability greater than $1-\mathfrak{N}\left(\mathrm{P}_{1}+2 \mathrm{P}_{0}\right)$ $\sim 1$ for every $z \in Z_{q}^{\prime}$ and $z=\partial c_{1}, c_{1} \in C_{q+1}$, there is a $c^{\prime} \in C_{q+1}^{\prime}$ such that $z=\partial c^{\prime}$.

Thus, $\mathcal{N}(\mathcal{P})$ is an adequate subcomplex of $\mathcal{N}(\mathcal{I})$ and therefore by Lemma $13, H(\mathcal{N}(\mathcal{P}))$ $\simeq H\left(\mathcal{N}(\mathcal{I})\right.$ ) with probability $1-\mathfrak{N}\left(\mathrm{P}_{1}+2 \mathrm{P}_{0}\right) \sim 1$. Moreover, by Claim $7 H(\mathcal{N}(\mathcal{I})) \simeq H(M)$ with probability $\sim 1-\mathfrak{N}\left(\mathrm{P}_{1}+\mathrm{P}_{2}\right)$. Thus, the joint probability for $H(\mathcal{N}(\mathcal{P})) \simeq H(M)$ is $\sim 1-\mathfrak{N}\left(2 \mathrm{P}_{1}\right.$ $\left.+\mathrm{P}_{2}+2 \mathrm{P}_{0}\right) \sim 1$.

\section{CONCLUSIONS}

What can we conclude about the Hauptvermutung? For an appropriate inextendible antichain $A \subset C$, our result states that there exists a wide range of thickenings $\mathrm{T}_{\mathrm{n}}(A), \mathrm{n} \gg 1$ for which $H_{q}(\mathcal{N}(\mathcal{P}))$ is isomorphic to $H_{q}(M)$ with high probability. Thus, it is crucial to be able to identify such an inextendible antichain in $C$. Assuming that it exists, and is unique, we have proven a weak form of the Hauptvermutung, i.e., if $\Phi: C \rightarrow M$ and $\Phi^{\prime}: C \rightarrow M^{\prime}$ are two possible faithful embeddings, then $H_{q}(M) \simeq H_{q}\left(M^{\prime}\right)$.

The problem therefore lies in how to identify an $A \subset C$ with which to perform such a test, i.e., one for which $\Phi(A) \subset M$ is such that the required separation of scales may be satisfied. Roughly speaking, for causets that embed into compact space-times, the largest convexity volume $\widetilde{v}$ might be expected for a Cauchy hypersurface with the smallest intrinsic and extrinsic curvatures. An $A$ that would lie in such a hypersurface might be obtained as follows: let $\mathrm{n}_{m}(A)$ be the smallest thickening scale of $A$ such that there exists an $m_{i} \in \mathrm{M} \subset \mathrm{T}_{\mathrm{n}_{m}}(A)$ with all of $A \subset I^{-}\left(m_{i}\right)$. For any causal set $C$ pick the inextendible antichain $A_{C}$ with the largest value of $\mathrm{n}_{m}(A)$. We can then perform the homology test on thickenings of $A_{C}$. This prescription should work reasonably for space-times for which there exists a scale $V \gg V_{c}$ for which the intrinsic and extrinsic curvatures are small.

However, it is not a priori clear that the required separation of scales is universal. For example, if $A$ satisfies this requirement in $(M, g)$, then it is possible that it does not in $\left(M^{\prime}, g^{\prime}\right)$, despite $\Phi^{\prime}$ being a faithful embedding. In such a case, our method does not allow us to infer a relationship between the homologies of $M$ and $M^{\prime}$.

In summary, a strict homology avatar of the Hauptvermutung would require us to pick an appropriate $A$ without any reference to the manifold. Further numerical work should help us understand how stable the homology calculation is as we vary over the inextendible antichains in a given causal set. ${ }^{14} \mathrm{~A}$ better understanding of how both intrinsic and extrinsic spatial geometries are encoded in a causal set should also help resolve this question ${ }^{21}$ and would, moreover, bring us closer to an understanding of how dynamical information is encoded in a causal set.

\section{ACKNOWLEDGMENTS}

The authors thank M. S. Narasimhan and John Ratcliffe for crucial help with the De RhamWeil theorem. They also thank Graham Brightwell, Fay Dowker, Joe Henson, Pawel Pilarczyk, and Rafael Sorkin for discussions. Figure 2 was generated using the CHOMP homology calculator package $^{22}$ and the Cactus framework. ${ }^{23}$ One of the authors (D.R.) was supported by the Marie Curie Research and Training Network ENRAGE (MRTN-CT-2004-005616). 


\section{APPENDIX: NOTATION}

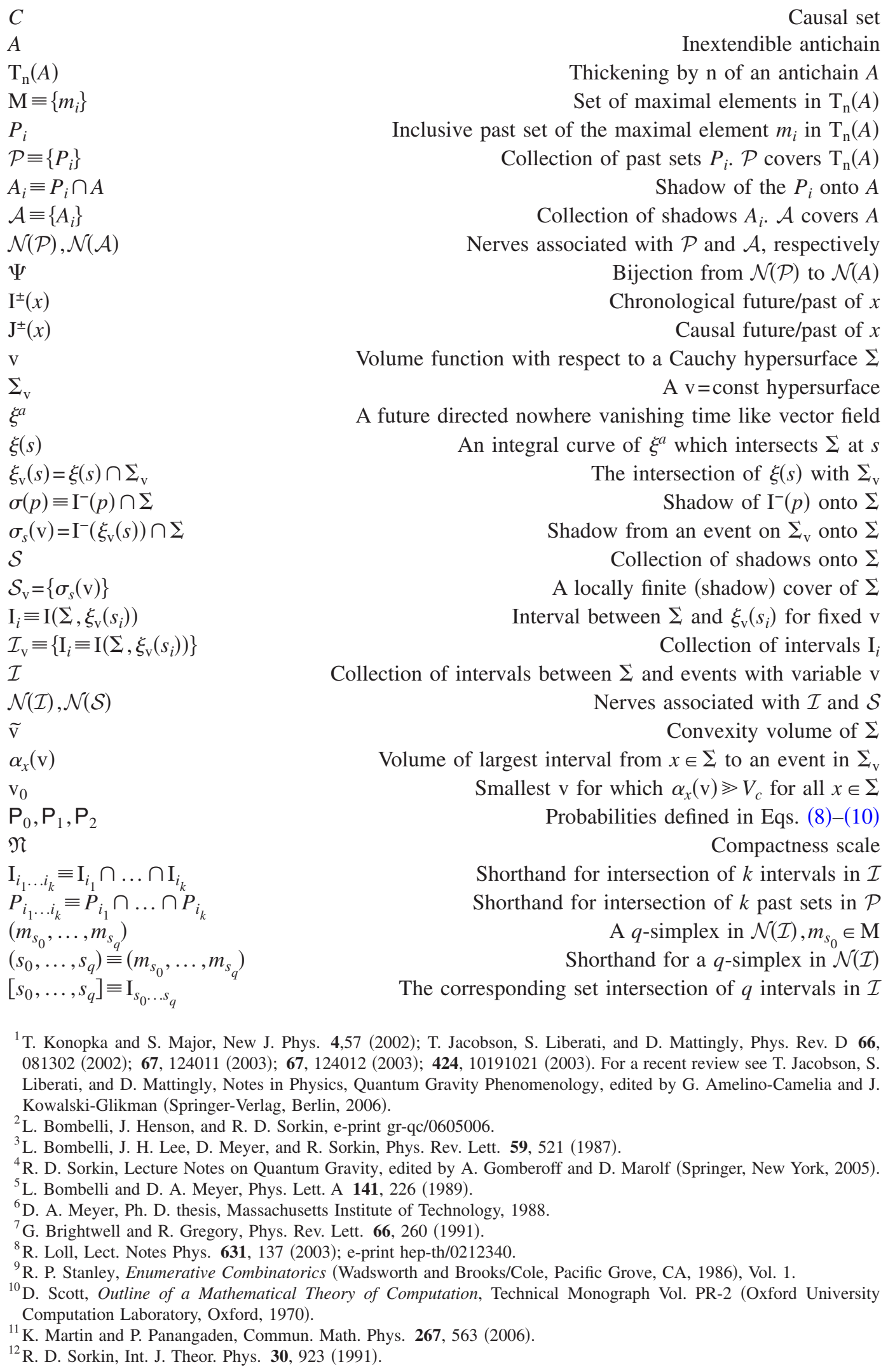

Causal set Inextendible antichain Thickening by $\mathrm{n}$ of an antichain $A$ Set of maximal elements in $\mathrm{T}_{\mathrm{n}}(A)$ Inclusive past set of the maximal element $m_{i}$ in $\mathrm{T}_{\mathrm{n}}(A)$ Collection of past sets $P_{i}$. $\mathcal{P}$ covers $\mathrm{T}_{\mathrm{n}}(A)$ Shadow of the $P_{i}$ onto $A$ Collection of shadows $A_{i}$. $\mathcal{A}$ covers $A$ Nerves associated with $\mathcal{P}$ and $\mathcal{A}$, respectively Bijection from $\mathcal{N}(\mathcal{P})$ to $\mathcal{N}(A)$ Chronological future/past of $x$ Causal future/past of $x$ Volume function with respect to a Cauchy hypersurface $\Sigma$ A $v=$ const hypersurface A future directed nowhere vanishing time like vector field An integral curve of $\xi^{a}$ which intersects $\Sigma$ at $s$ The intersection of $\xi(s)$ with $\Sigma_{\mathrm{v}}$ Shadow of $\mathrm{I}^{-}(p)$ onto $\Sigma$ Shadow from an event on $\Sigma_{\mathrm{v}}$ onto $\Sigma$ Collection of shadows onto $\Sigma$ A locally finite (shadow) cover of $\Sigma$ Interval between $\Sigma$ and $\xi_{\mathrm{v}}\left(s_{i}\right)$ for fixed $\mathrm{v}$ Collection of intervals $\mathrm{I}_{i}$ Collection of intervals between $\Sigma$ and events with variable $\mathrm{v}$ Nerves associated with $\mathcal{I}$ and $\mathcal{S}$ Convexity volume of $\Sigma$ Volume of largest interval from $x \in \Sigma$ to an event in $\Sigma_{\mathrm{v}}$ Smallest $\mathrm{v}$ for which $\alpha_{x}(\mathrm{v}) \gg V_{c}$ for all $x \in \Sigma$ Probabilities defined in Eqs. (8)-(10) Compactness scale Shorthand for intersection of $k$ intervals in $\mathcal{I}$ Shorthand for intersection of $k$ past sets in $\mathcal{P}$ A $q$-simplex in $\mathcal{N}(\mathcal{I}), m_{s_{0}} \in \mathrm{M}$ Shorthand for a $q$-simplex in $\mathcal{N}(\mathcal{I})$ The corresponding set intersection of $q$ intervals in $\mathcal{I}$

${ }^{1}$ T. Konopka and S. Major, New J. Phys. 4,57 (2002); T. Jacobson, S. Liberati, and D. Mattingly, Phys. Rev. D 66, 081302 (2002); 67, 124011 (2003); 67, 124012 (2003); 424, 10191021 (2003). For a recent review see T. Jacobson, S. Liberati, and D. Mattingly, Notes in Physics, Quantum Gravity Phenomenology, edited by G. Amelino-Camelia and J. Kowalski-Glikman (Springer-Verlag, Berlin, 2006).

${ }^{2}$ L. Bombelli, J. Henson, and R. D. Sorkin, e-print gr-qc/0605006.

${ }^{3}$ L. Bombelli, J. H. Lee, D. Meyer, and R. Sorkin, Phys. Rev. Lett. 59, 521 (1987).

${ }^{4}$ R. D. Sorkin, Lecture Notes on Quantum Gravity, edited by A. Gomberoff and D. Marolf (Springer, New York, 2005).

${ }^{5}$ L. Bombelli and D. A. Meyer, Phys. Lett. A 141, 226 (1989).

${ }^{6}$ D. A. Meyer, Ph. D. thesis, Massachusetts Institute of Technology, 1988.

${ }^{7}$ G. Brightwell and R. Gregory, Phys. Rev. Lett. 66, 260 (1991).

${ }^{8}$ R. Loll, Lect. Notes Phys. 631, 137 (2003); e-print hep-th/0212340.

${ }^{9}$ R. P. Stanley, Enumerative Combinatorics (Wadsworth and Brooks/Cole, Pacific Grove, CA, 1986), Vol. 1.

${ }^{10} \mathrm{D}$. Scott, Outline of a Mathematical Theory of Computation, Technical Monograph Vol. PR-2 (Oxford University Computation Laboratory, Oxford, 1970).

${ }^{11}$ K. Martin and P. Panangaden, Commun. Math. Phys. 267, 563 (2006).

${ }^{12}$ R. D. Sorkin, Int. J. Theor. Phys. 30, 923 (1991). 
${ }^{13}$ S. Major, D. Rideout, and S. Surya, Class. Quantum Grav. 23, 4743 (2006).

${ }^{14}$ S. Major, D. Rideout, and S. Surya (unpublished).

${ }^{15}$ S. W. Hawking and G. F. R. Ellis, The Large Scale Structure of Space-time (Cambridge University Press, Cambridge, 1973).

${ }^{16}$ S. W. Hawking and R. R. Sachs, Commun. Math. Phys. 35, 287 (1974).

${ }^{17}$ P. Petersen, Graduate Texts in Mathematics (Springer-Verlag, New York, 1998).

${ }^{18}$ J. J. Rotman, An Introduction to Algebraic Topology (Springer-Verlag, New York, 1993).

${ }^{19}$ A. Weil, Comment. Math. Helv. 26, 119 (1952); G. De Rham, Ann. Inst. Fourier 2, 51 (1950).

${ }^{20}$ G. Perelman, e-print org:math/0211159.

${ }^{21}$ S. Major, D. Rideout, and S. Surya (unpublished).

${ }^{22}$ CHOMP, http://www.math.gatech.edu/chomp/

${ }^{23}$ T. Goodale, G. Allen, G. Lanfermann, J. Masso, T. Radke, E. Seidel, and J. Shalf, The cactus framework and toolkit: Design and applications, in Vector and Parallel Processing-VECPAR 2002, 5th International Conference (Springer, 2003). 